\title{
稳定嗍烷加合物在有机嗍化物合成中的应用研究进展
}

\author{
杨吉民 $a$ 李子奇 $a$ 朱守非*, $a, b$ \\ ( ${ }^{a}$ 南开大学化学学院 元素有机化学研究所 元素有机化学国家重点实验室 天津 300071) \\ ( ${ }^{b}$ 天津化学化工协同创新中心 天津 300071 )
}

\begin{abstract}
摘要 有机嗍化合物在合成化学、材料科学、生命健康等领域都有广泛应用，因此有机嗍化合物的合成一直是研究热 点. 目前, 催化 $\mathrm{C}-\mathrm{B}$ 键形成反应通常使用联嗍酸频哪醇酯 $\left(\mathrm{B}_{2} \mathrm{Pin}_{2}\right)$ 、频哪醇硓烷(HBPin)、儿茶酚硼烷(HBCat) 等作为 喼试剂. 相比于传统的喼试剂, 硼烷与胺、膦或 N-杂环卡宾等强 Lewis 碱的加合物(统称为稳定硼烷加合物)具有易于合 成、稳定性高、易操作等特点, 其作为嗍试剂参与的有机嗍化合物的合成最近受到越来越多的关注, 已被成功用于烯 (炔)烃的嗍氢化、 $\mathrm{C}-\mathrm{H}$ 键嗍化、卡宾对 $\mathrm{B}-\mathrm{H}$ 键的插入、嗍自由基串联环化、取代等反应中，为有机嗍化合物的合成 提供了新的思路和方法. 以反应类型为线索, 系统综述了稳定嗍烷加合物在有机嗍化物合成中的应用研究进展.
\end{abstract}

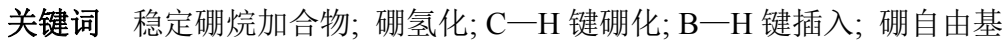

\section{Progresses on the Application of Stable Borane Adducts in the Synthesis of Organoborons}

\author{
Yang, $\operatorname{Jimin}^{a}$ \\ Li, Ziqi ${ }^{a}$ \\ Zhu, Shoufei*,a,b \\ ( ${ }^{a}$ State Key Laboratory and Institute of Elemento-Organic Chemistry, College of Chemistry, \\ Nankai University, Tianjin 300071) \\ ( ${ }^{b}$ Collaborative Innovation Center of Chemical Science and Engineering, Tianjin 300071)
}

\begin{abstract}
Organoboron compounds are wildly used in organic synthesis, materials science, life and health science, etc. The development of synthetic methodologies of organoborons has therefore gained intense attention nowadays. Typically, Bis(pinacolato)diboron $\left(\mathrm{B}_{2} \mathrm{Pin}_{2}\right)$, pinacolborane (HBpin) and catecholatoborane (HBCat) are predominantly used as boron reagents in catalytic $\mathrm{C}-\mathrm{B}$ bond forming reactions. Different from the above traditional boron reagents, borane adducts with strong Lewis bases, such as amines, phosphines, and N-heterocyclic carbenes, are promising boron reagents because of their readily accessibility, relatively high stability, and easy operation. Moreover, the different chemical properties of these stable borane adducts towards the traditional boron reagents provide possibilities for development of new $\mathrm{C}-\mathrm{B}$ bond formation reactions. The applications of the stable borane adducts as terminal boron reagents in hydroboration of alkenes or alkynes, $\mathrm{C}-\mathrm{H}$ bond borylation, carbene insertion into $\mathrm{B}-\mathrm{H}$ bonds, cascade cyclization initiated by boryl radicals and substitutions, which provide new methods for the preparation of organoborons are reviewed in this paper.

Keywords stable borane adduct; hydroboration; $\mathrm{C}-\mathrm{H}$ bond borylation; $\mathrm{B}-\mathrm{H}$ bond insertion; boryl radical
\end{abstract}

硼烷与胺、膦或氮杂环卡宾化合物等强 Lewis 碱形 成的稳定配合物分别叫做胺嗍烷加合物、膦硼烷加合 物、吡啶嗍烷加合物或氮杂环卡宾(NHC)嗍烷加合物 (Scheme 1). 这些加合物在成键模式上与弱 Lewis 碱(如 醚、硫醚等)与硼烷形成的配合物相似，都是 Lewis 碱的
孤对电子填充到硼烷的空 $\mathrm{p}$ 轨道上成键, 但是其稳定性 更高，可以统称为稳定硼烷加合物，而且与后者在性质 上也表现出很大的不同. 由于硼烷的空 $\mathrm{p}$ 轨道被 Lewis 碱的孤对电子所填充, 所以相应的硼烷加合物具有较高 的稳定性，通常对水和空气都不敏感. 另一方面，由于

\footnotetext{
* Corresponding author. E-mail: sfzhu@nankai.edu.cn Received May 23, 2017; revised July 18, 2017; published online August 9, 2017.

Project supported by the National Natural Science Foundation of China (Nos. 21625204, 21421062, 21290182), the National Basic Research Program of China (973 Program, No. 2012CB821600), the "111" Project of the Ministry of Education of China (No. B06005), and the National Program for Support of Top-notch Young Professionals.

国家自然科学基金(Nos. 21625204, 21421062, 21290182)、国家重点基础研究发展计划(973 项目, No. 2012CB821600)、教育部“111 计划”(No. B06005) 和国家特支计划资助项目.
} 
配位键的存在，使得嗍烷加合物中的 Lewis 碱和嗍烷之 间形成了推拉电子的结构, 硼中心由 $\mathrm{sp}^{2}$ 杂化变为类 $\mathrm{sp}^{3}$ 杂化, 同时由平面结构变为类四面体结构, 嗍中心的电 子云密度明显提高. 正是由于稳定硼烷加合物这种独特 的电子结构, 使其具有不同于硼烷和硼酸酯的反应性, 同时还具有较高的稳定性, 在很多领域都有应用. 胺硼 烷加合物 ${ }^{[1]}$ 是被研究得较多的一类硼试剂, 它在较低温 度下可以用作温和的还原剂、储氢试剂、配体等. 较高 温度下胺嗍烷可以释放出自由硼烷表现出硼烷的性质, 硼烷释放的温度可以被不同结构的胺控制, 所以胺嗍烷 也是一种可控释放硼烷的化合物. 此外, 胺硼烷加合物 作为氢给体被成功用于多种不饱和化合物的氢化反应 中 $^{[2]}$. 吡啶嗍烷加合物可以看成一类特殊的胺嗍烷加合 物, 两者性质比较类似. 膦嗍烷加合物 ${ }^{[1]}$ 通常比胺嗍烷 更加稳定, 更难以分解, 它们易于制备, 被广泛用于有 机磷化学, 如有机磷的分离、保护或活化. 氮杂环卡宾 嗍烷加合物 ${ }^{[3]}$ 相对更加稳定, 近些年对于它们的研究逐 渐得到关注，展现出了不同于胺和膦嗍烷加合物的性 质，其作为还原剂、反应试剂、引发剂等在有机合成化 学和高分子化学中展现出了巨大的潜力.
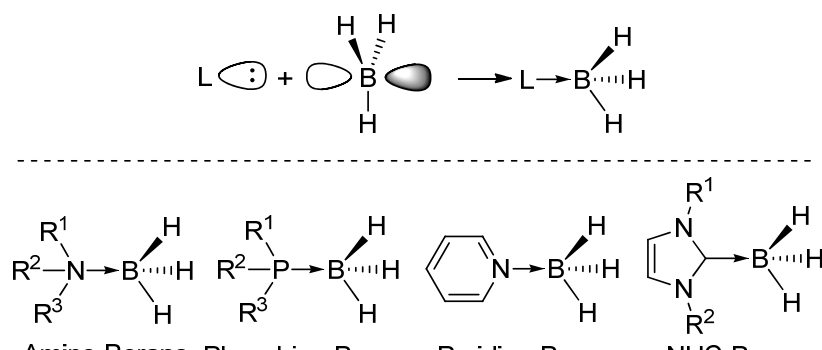

Amine-Borane Phosphine-Borane Pyridine-Borane NHC-Borane

图式 1 稳定硼烷加合物的成键原理及主要类型

Scheme 1 Bond-forming principle and main classes of stable borane adducts

有机硼化合物在合成化学、材料科学、医药等领域 都有广泛应用, 因此有机硼化合物的合成一直是研究热 点 ${ }^{[4]}$. 目前, 催化碳-嗍键形成反应通常使用联硼酸频哪 醇酯 $\left(\mathrm{B}_{2} \mathrm{Pin}_{2}\right)$ 、频哪醇硼烷(HBPin)、儿茶酚硼烷(HBCat) 等作为硼试剂 ${ }^{[5]}$. 一方面这些硼试剂通常比较容易水解, 稳定性欠佳; 另一方面, 这些硼试剂制备相对复杂 ${ }^{[6]}$, 如 $\mathrm{B}_{2} \mathrm{Pin}_{2}$ 通常以强腐蚀性的三溴化硼作为起始原料, 通 过多步转化制得, 产生较多污染性废物. 近年来, 稳定 嗍烷加合物作为一种易得、稳定的硼试剂逐渐被用到了 有机嗍化合物的合成中, 并展现出一些独特优势, 受到 了越来越多的关注. 本文根据反应类型的不同, 对稳定 嗍烷加合物参与的有机嗍化合物的合成进行了系统的 梳理和总结, 阐述了反应的机理, 并对该领域未来发展 进行了展望.

\section{1 稳定硼烷加合物的合成}

稳定硼烷加合物的合成非常方便(Scheme 2): 实验 室合成一般采用胺、膦或氮杂环卡宾与 $\mathrm{BH}_{3} \cdot \mathrm{THF}$ 或 $\mathrm{BH}_{3} \cdot \mathrm{SMe}_{2}$ 反应 ${ }^{[7]}$, 取代弱 Lewis 碱生成稳定的硼烷加合 物; 胺或膦的酸盐与 $\mathrm{NaBH}_{4}$ 或 $\mathrm{LiBH}_{4}$ 反应也可以生成相 应的嗍烷加合物 ${ }^{[8]}$, 工业上一般采用此方法大规模制备; 此外在适当反应条件下，胺硼烷加合物或膦硼烷加合物 可以与另一分子胺、膦或氮杂环卡宾发生交换反应生成 相应的硼烷加合物 ${ }^{[9]}$.

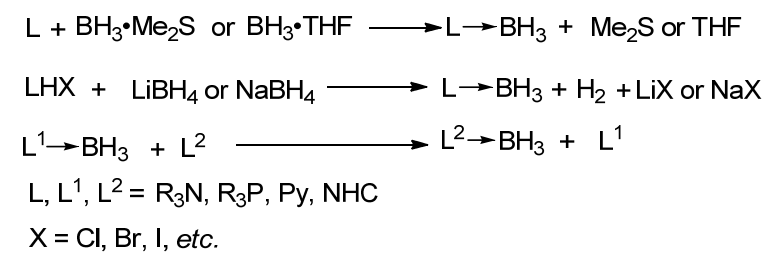

图式 2 稳定嗍烷加合物的合成方法

Scheme 2 Synthetic methods of stable borane adducts

\section{2 烯(炔)的硼氢化反应}

烯(炔)的硼氢化反应是合成有机嗍化合物的常用方 法, 该反应通常使用 $\mathrm{BH}_{3} \cdot \mathrm{THF} 、 \mathrm{BH}_{3} \cdot \mathrm{SMe}_{2} 、 9$-borabicyclo[3.3.1]nonane (9-BBN)、HBpin 和 HBCat 等高活性 的嗍试剂. 稳定硼烷加合物在室温下通常不能与不饱和 烃发生硼氢化反应. 在早期的研究中，通常在较高的温 度下硼烷加合物发生解离, 解离后的自由硼烷再与不饱 和烃发生硼氢化反应 ${ }^{[10]}$. 近年来，一些活化剂或催化剂 促进的嗍烷加合物与烯(炔)的嗍氢化反应开始见诸报 道.

\section{1 碘或酸促进的嗍氢化反应}

2003 年, Vedejs 等 ${ }^{[11]}$ 以 $\mathrm{I}_{2}$ 为活化试剂(50 mol\%)实现 了首例高烯丙基胺 1 的嗍烷加合物分子内嗍氢化反应, 生成的环状有机嗍 $\mathbf{2 a}$ 和 $\mathbf{2 b}$ 经氧化得到相应的醇 $3 \mathrm{a}$ 和 3b，区域选择性主要受烯烃上的取代基 $\mathrm{R}$ 控制(Scheme $3)$. 该反应用催化量的 $I_{2}(10 \mathrm{~mol} \%)$ 同样可以顺利进行, 收率和选择性都较好.

机理研究认为(Scheme 4): 当体系中没有活化剂的 时候, 如果要发生嗍氢化反应, 需要双键进攻嗍原子, 经由过渡态 5 断裂 $\mathrm{N}$ - B 配键生成 $\mathbf{6}$ 才能完成——然而 这一过程在轨道匹配性上是不利的 ${ }^{[12]}$, 因而不能发生 (Path a); 当体系加入活化试剂 $\mathrm{I}_{2}$ 之后, 可以形成带有不 稳定 B-I 键的中间体 7, 随后双键对硼原子进攻, 经由 共价型过渡态 8 或离子型过渡态 $\mathbf{9}$, 生成硼烷与烯烃结 合的中间体 10, 随后发生硼氢化得到目标产物(Path b 和 Path c).

三氟甲磺酸能够有效地促进高烯丙基膦硼烷加合 


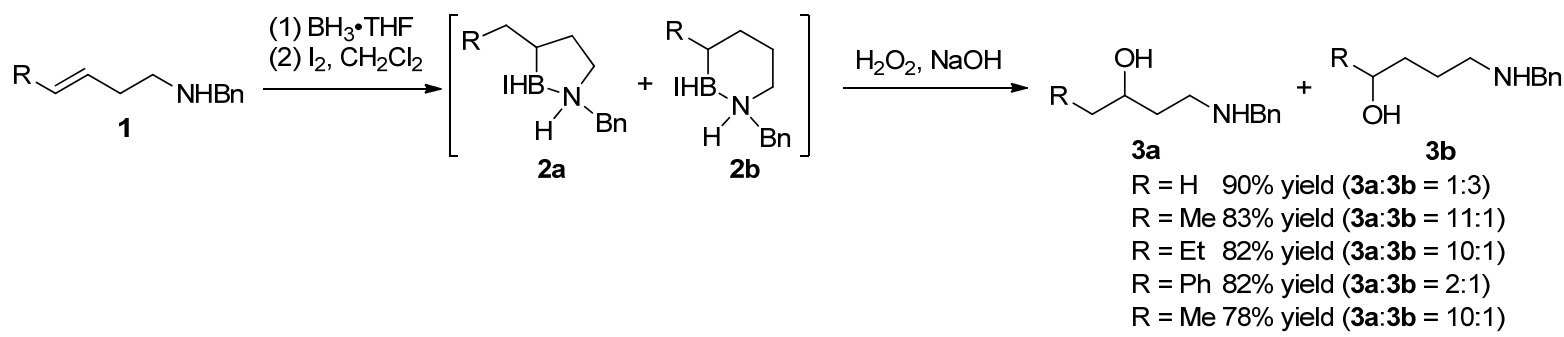

图式 3 碘促进的高烯丙基胺一嗍烷加合物的分子内嗍氢化反应

Scheme 3 Iodine-promoted hydroboration of homoallylic amine-borane adducts

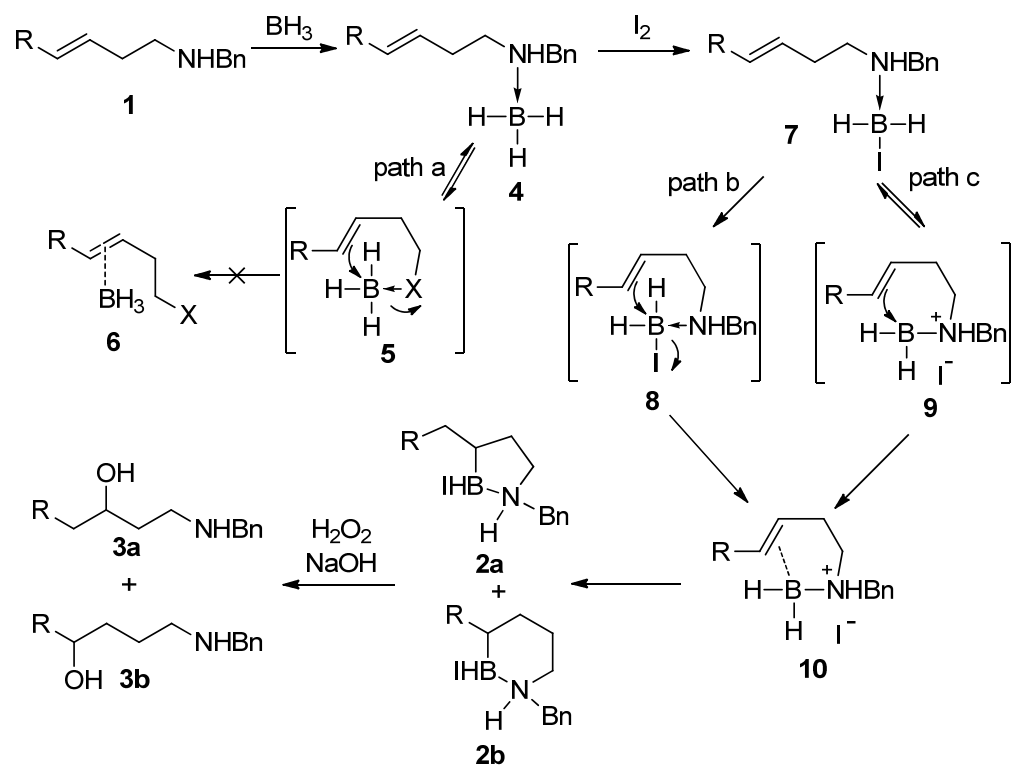

图式 4 高烯丙基胺嗍烷加合物的分子内嗍氢化反应可能机理

Scheme 4 Proposed mechanisms for the intramolecular hydroboration of homoallylic amine-borane adducts

物 11 的硼氢化反应, 生成磷一嗍杂环化合物 $12 \mathrm{a}$ 和 $12 \mathrm{~b}$, 经氧化后生成相应的醇 $13 \mathrm{a}$ 和 $13 \mathrm{~b}$, 反应的区域选择性 比相应的高烯丙胺嗍烷加合物高(Scheme 5). 和 $\mathrm{I}_{2}$ 的作 用相似，三氟甲磺酸通过和碀烷反应生成活性更高的离 子型嗍烷中间体, 促进了烯烃和嗍烷的作用, 进而完成 了嘲氢化反应.

2005 年, 以 $I_{2}$ 为活化剂, Vedejs 等 ${ }^{[13]}$ 实现了烯烃和 吡啶嗍烷加合物的分子间喼氢化反应(Scheme 6). 一系 列控制实验表明, 反应可能机理为: 吡啶硼烷加合物与 碘作用生成吡啶碘硼烷加合物 14, 随后双键对其进行
类 $\mathrm{S}_{\mathrm{N}} 2$ 亲核进攻生成离子对中间体 15 , 最后经历四中心 环状过渡态发生硼氢化反应生成 16, 再经氧化得到相 应的醇 17.

利用这个策略他们同样实现了嗍烷加合物与炔烃 的分子间硼氢化反应，生成的烯基嗍化合物 $18 \mathrm{a}$ 和 $18 \mathrm{~b}$ 经氧化后生成相应的酮 19a 和 19b. 该反应的区域选择 性受立体位阻影响，当炔烃两端取代基大小相似时，区 域选择性很差(Scheme 7).

$\mathrm{I}_{2}$ 活化吡啶硼烷加合物和烯烃的嗍氢化反应在合成 中有很好的潜在应用价值. 嗍氢化产物除了上文所展示

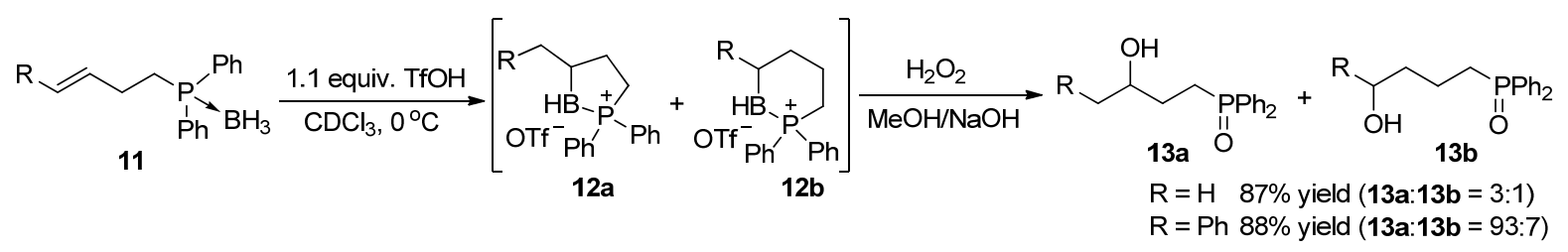

图式 $5 \mathrm{TfOH}$ 促进的高烯丙基膦硼烷加合物的分子内嗍氢化反应

Scheme 5 TfOH-promoted intramolecular hydroboration of homoallylic phosphine-borane adducts 


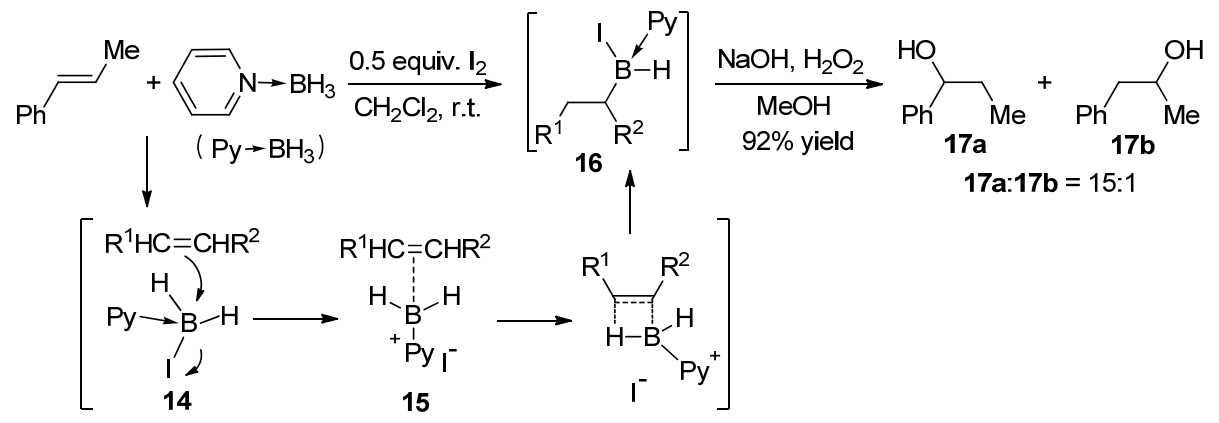

图式 $6 \mathrm{I}_{2}$ 促进烯烃和吡啶硼烷加合物的分子间硼氢化反应

Scheme $6 \mathrm{I}_{2}$-promoted intermolecular hydroboration of alkenes with pyridine borane adducts

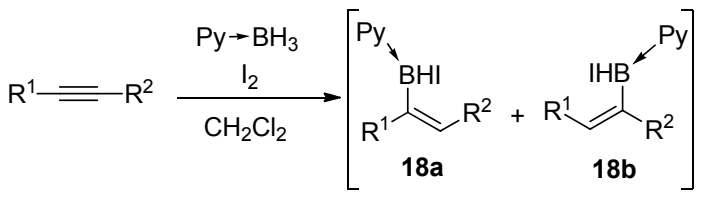

\begin{tabular}{|c|c|c|c|c|}
\hline$\frac{\mathrm{NaOH}, \mathrm{H}_{2} \mathrm{C}}{\mathrm{MeOH}}$ & & 19a & & $R^{2}$ \\
\hline & $\mathrm{R}^{1}$ & $\mathrm{R}^{2}$ & Yield/\% & 19a:19b \\
\hline & $\mathrm{Ph}$ & Me & 64 & 15:1 \\
\hline & $\mathrm{Ph}$ & Et & 66 & $10: 1$ \\
\hline & $\mathrm{Me}$ & $n-\mathrm{C}_{5} \mathrm{H}_{11}$ & 61 & $1.5: 1$ \\
\hline & $\mathrm{Me}$ & $c-\mathrm{C}_{6} \mathrm{H}_{11}$ & 64 & $3: 1$ \\
\hline
\end{tabular}

图式 $7 \mathrm{I}_{2}$ 促进炔烃和吡啶嗍烷加合物的分子间嗍氢化反应 Scheme $7 \quad I_{2}$-promoted intermolecular hydroboration of alkynes with pyridine borane adduct

的氧化为醇, 还可以进一步转化为在有机合成中常用的 烷基频哪醇硼酸酯和三氟硼酸钾等有机硣试剂(Scheme $8)^{[14]}$.

最近, Shenvi 等以 $I_{2}$ 活化氨基硼烷加合物的分子内 嗍氢化反应为基础, 结合一个串联的烷基迁移反应, 方 便地构筑了 $\mathrm{N}$ - 杂双环结构, 并成功合成了 Indolizidine ${ }^{[15]}$ 和 Lepadiformine ${ }^{[16]}$ 等天然产物 (Scheme 9).

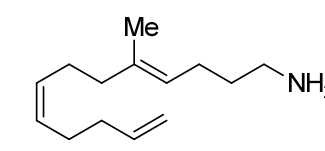

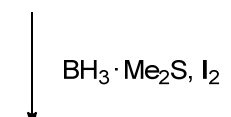

$$
\mathrm{H}_{2}
$$

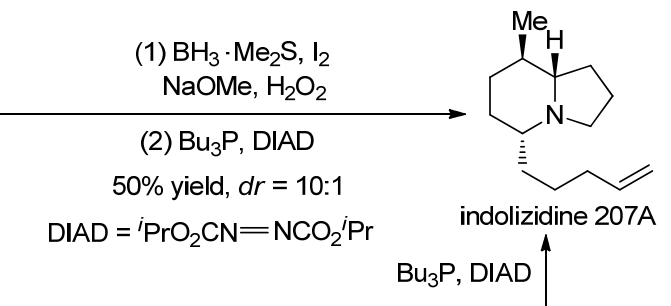<smiles>C=CCCC[C@H]1CCCCC12CCCC[P+]21NCCCC1C</smiles>

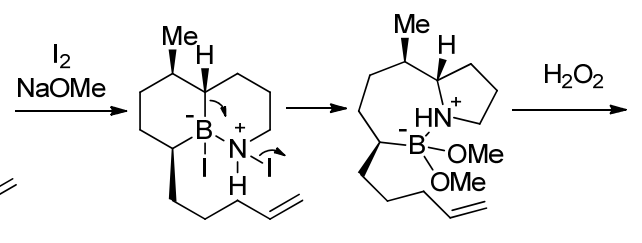

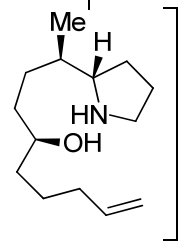

图式 9 氨基硼烷加合物的嗍氢化用于天然产物的合成

Scheme 9 Applications of hydroboration of amine-borane adducts in natural product synthesis 


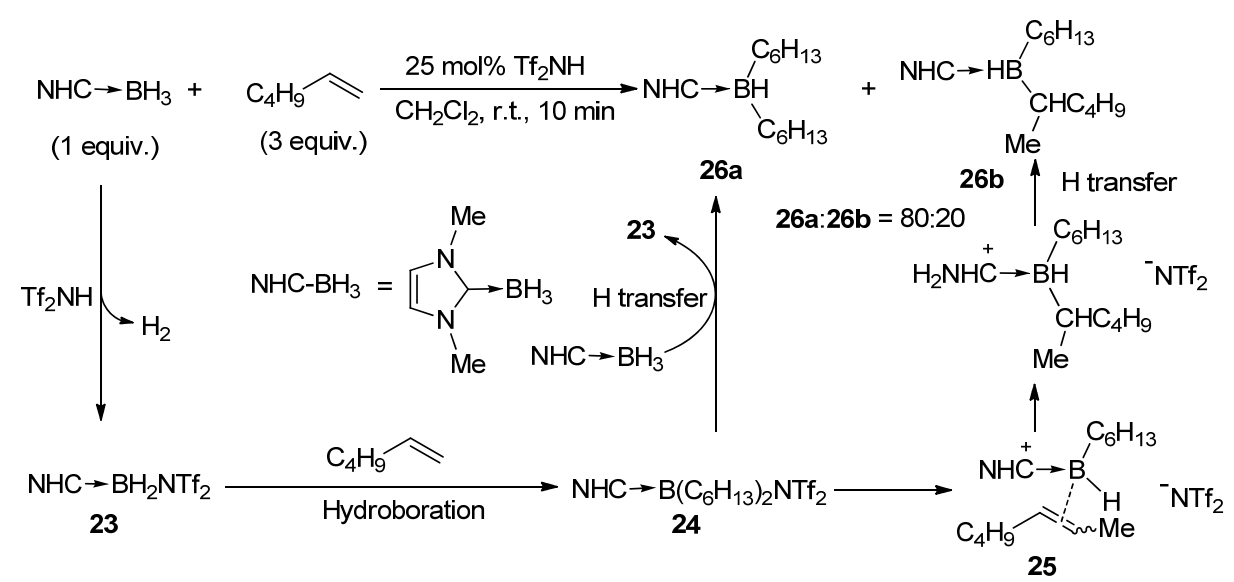

图式 $10 \mathrm{Tf}_{2} \mathrm{NH}$ 催化的烯烃和氮杂环卡宾嗍烷加合物的嗍氢化反应

Scheme $10 \mathrm{Tf}_{2} \mathrm{NH}$-catalyzed hydroboration of alkenes with $\mathrm{NHC}-\mathrm{BH}_{3}$

$\mathrm{NHC}-\mathrm{BH}_{3}$ 发生氢转移, 生成目标产物 26a, 同时重新生 成活性催化剂 23. 因为活性中间体 $\mathbf{2 4}$ 不含 $\mathrm{B}-\mathrm{H}$ 键, 无 法进行硼氢化反应, 所以卡宾硼烷加合物虽然含有三个 $\mathrm{B}-\mathrm{H}$ 键, 但是只能得到二次硼氢化的产物. 虽然该催 化体系可以用于端烯和内烯的嗍氢化, 但是由于嗍鎓阳 离子容易在烷基链上迁移(经由中间体 25), 因此会生成 迁移产物 26b, 造成反应的区域选择性很难控制.

随后, Curran 等 ${ }^{[18]}$ 使用催化量的 $\mathrm{I}_{2}$ 实现了 $\mathrm{NHC}^{-\mathrm{BH}_{3}}$ 和烯烃的磁氢化反应, 给出中等或较高的收率 (表 1$)$. 与 $\mathrm{Tf}_{2} \mathrm{NH}$ 催化剂相比, $\mathrm{I}_{2}$ 催化的硼氢化具有更广泛的底物 适用范围, 可以生成稳定的可通过柱层析分离的单烷基 嗍烷加合物. 此方法也可用于嗍杂环化合物的合成, 比 如与 1,5 -环辛二烯反应合成硼杂双环化合物 $\mathbf{2 7}$, 而通过 分子内的嗍氢化反应可以合成硼杂并环化合物 28 (Scheme 11).

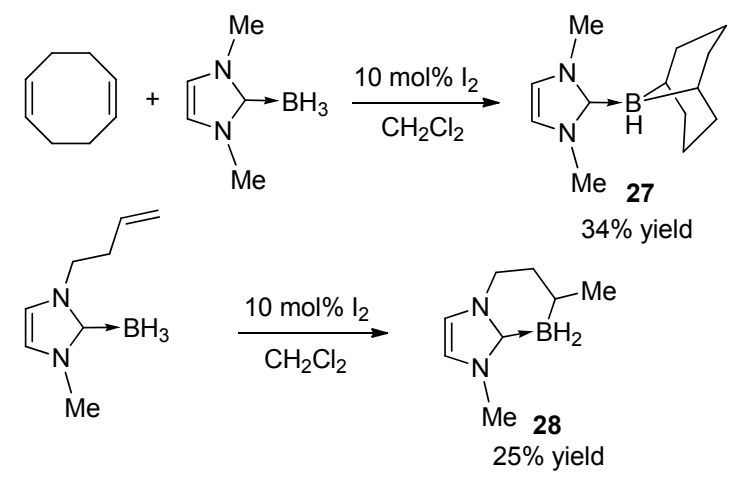

图式 11 环状氮杂环卡宾嗍烷的合成

Scheme 11 Synthesis of cyclic NHC-borane adducts

Curran 等 ${ }^{[19]}$ 提出了如 Scheme 12 所示反应机理, NHC- $\mathrm{BH}_{3}$ 与 $\mathrm{I}_{2}$ 作用生成共价型的嗍碘化合物 29, 该化 合物可以在溶液中长时间保存, 但对水、醇和烯烃等中 性亲核试剂不稳定. 嗍碘化合物 29 在反应中起到了催 化剂的作用，它被烯烃进攻生成中间体或过渡态 $\mathbf{3 0}$, 随
表 $1 \mathrm{I}_{2}$ 催化的烯烃和氮杂环卡宾硼烷加合物的硼氢化反应 Table 1 I -catalyzed hydroboration of alkenes with $\mathrm{NHC}^{-\mathrm{BH}_{3}}$

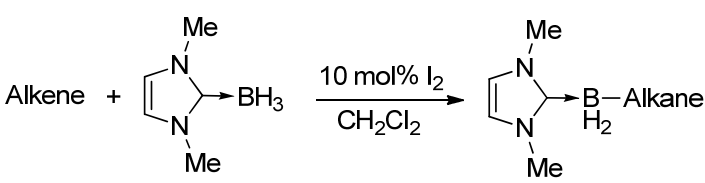<smiles>CC(C)=CC(C)=CC=C(C)C(C)=C(C)C</smiles>

后发生快速嗍氢化生成中间体 $\mathbf{3 1}$, 接下来与硼中心相 


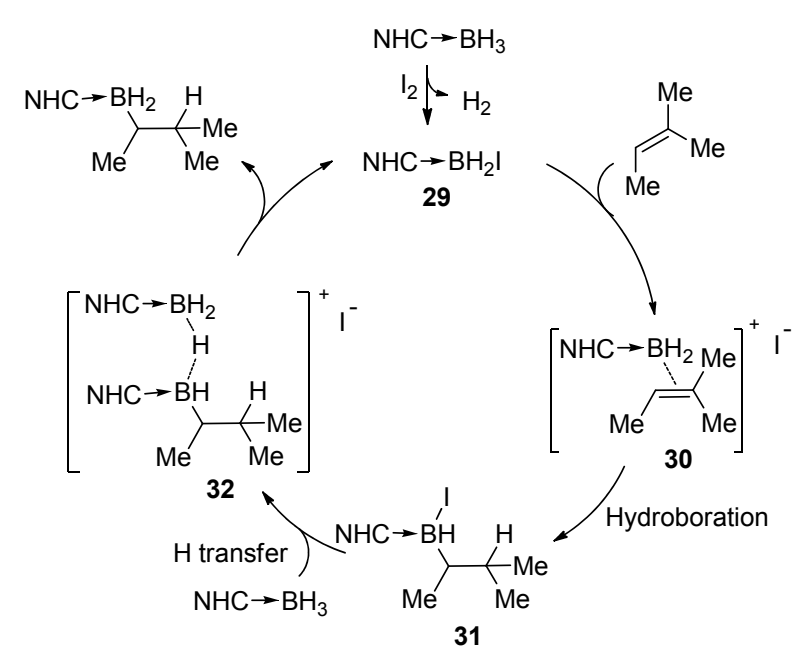

图式 $12 \mathrm{I}_{2}$ 催化的烯烃和氮杂环卡宾嗍烷加合物的硼氢化反 应可能机理

Scheme 12 Proposed mechanism of $\mathrm{I}_{2}$-catalyzed hydroboration of alkenes with $\mathrm{NHC}_{-} \mathrm{BH}_{3}$

连的碘再次被 $\mathrm{NHC}-\mathrm{BH}_{3}$ 上的氢取代, 经由桥连中间体 32 生成硼氢化产物, 同时重新生成活性催化剂 29, 完成 催化循环.

2013 年, Curran 等 ${ }^{[20]}$ 报道了 $\mathrm{Tf}_{2} \mathrm{NH}$ 或 $\mathrm{I}_{2}$ 促进的 $\mathrm{NHC}-\mathrm{BH}_{3}$ 和硅基取代炔烃的硼氢化反应(Scheme 13). 比较有意思的是, 当底物为二硅基取代的炔烃时, 生成 的是伴随着硅基迁移的 1,1-硼氢化产物 33. 该反应对于 烷基硅基炔烃底物选择性较差, 生成的主要是多个二次 嗍氢化产物的异构体 34a 34c, 对于苯基硅基炔烃底 物主要得到单次嗍氢化的产物 35 .

\section{2 金属催化的嗍氢化反应}

相比于碘或酸促进的嗍烷加合物的硼氢化反应，金 属催化的嗍烷加合物嗍氢化反应成功的例子较少. 2011 年, Weller 等 ${ }^{[21]}$ 以 $\mathrm{Rh}\left(\mathrm{P}^{i} \mathrm{Bu}_{2}{ }^{t} \mathrm{Bu}\right)_{2} \mathrm{BAr} \mathrm{F}_{\mathrm{F}}$ 为催化剂实现了三 甲胺硼烷加合物与叔丁基乙烯 36 的嗍氢化反应(Scheme 14). 他们认为反应机理为: 催化剂与三甲胺嗍烷生成 中间体 38, 随后铑对 $\mathrm{B}-\mathrm{H}$ 键进行氧化加成生成中间体 39, 再对烯烃进行迁移插入生成中间体 40, 接下来进行 还原消除生成中间体 41, 最后与三甲胺硼烷发生交换 生成反马氏路氢化的产物 $\mathbf{3 7}$, 完成催化循环. 中间体 41 得到了单晶衍射的确认. 不过此催化体系只适用于叔丁 基乙烯的底物。在随后的研究中, 他们发现 $\mathrm{Rh}$ (xantphos)BAr ${ }_{\mathrm{F}}{ }^{[22]}$ 也可以催化此类反应, 并且进行了 详细的机理实验, 认为还原消除的过程是反应的决速 步.

2012 年, Parrain 等 ${ }^{[23]}$ 以离子型的 Rh(I) 和手性双膦 配体作催化剂实现了金属催化的氮杂环卡宾嗍烷加合 物的分子内嗍氢化反应(Eq. 1). 他们以烯丙基取代的氮 杂环卡宾嗍烷加合物 42 为底物, 能以最高 $94 \%$ 的收率

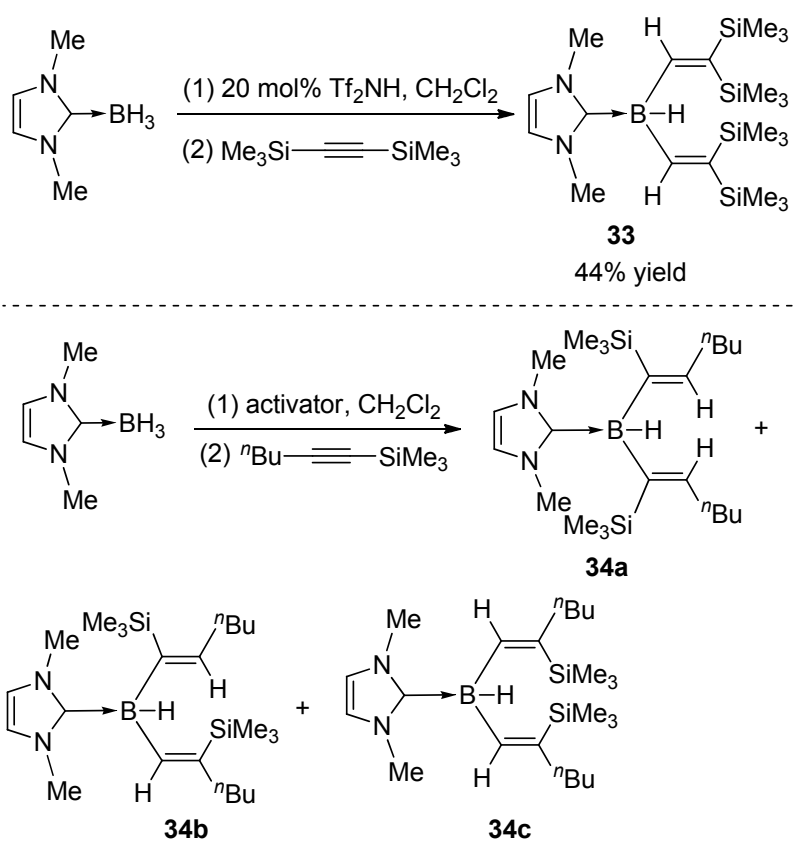

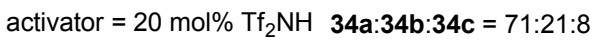
activator $=10 \mathrm{~mol} \% \mathrm{I}_{2} \quad 3 \mathbf{3} \mathbf{a}: \mathbf{3 4 b}: \mathbf{3 4 c}=86: 8: 6$

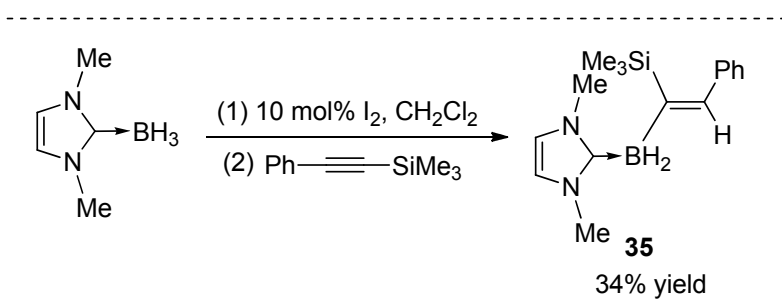

图式 $13 \mathrm{Tf}_{2} \mathrm{NH}$ 或 $\mathrm{I}_{2}$ 促进的硅基取代的炔烃和氮杂环卡宾硼 烷加合物的嗍氢化反应

Scheme $13 \mathrm{Tf}_{2} \mathrm{NH} / \mathrm{I}_{2}$-promoted hydroboration of silylsubstituted alkynes with $\mathrm{NHC}-\mathrm{BH}_{3}$

和 $98.4 \%$ ee 的对映选择性得到五元环状含喼化合物 43 .

2014 年，史晓东等 ${ }^{[24]}$ 报道了金催化的分子内的炔 烃喼氢化反应(Scheme 15). 他们采用氰基硼烷胺加合 物 44 和三氮唑(TA)稳定的 $\mathrm{Au}(\mathrm{I})$ 催化剂, 避免了催化剂 的失活，使得反应能顺利发生，以中等或较高的收率合 成了一系列含氮硼杂环化合物 45. 他们推测反应机理 为金催化剂活化炔烃(经中间体 46), 随后嗍烷加合物的 负氢对炔烃进攻生成烯基金中间体 47 进而生成目标产 物. 产物 45 中的氧基可以和 $\mathrm{LiAlH}_{4}$ 或 $\mathrm{PhMgBr}$ 发生取 代反应，分别生成新的氮硼杂环化合物 48 或 49.

2016 年，史晓东等 ${ }^{[25]}$ 使用金络合物和 $\mathrm{Cu}(\mathrm{OTf})_{2}$ 作 为催化剂实现了含炔基结构的氨基硼烷加合物 $\mathbf{5 0}$ 的分 子内硼氢化反应，合成了六元环状硼胺化合物 51 (Eq. $2)$. 当不加入 $\mathrm{Cu}(\mathrm{OTf})_{2}$ 时只能得到较差的转化率和选择 性. 他们认为 Lewis 酸催化剂 $\mathrm{Cu}(\mathrm{OTf})_{2}$ 可以协助亚磷酸 酯-三氮唑- $\mathrm{Au}$ 络合物催化剂中三氮唑的离去, 从而生 成活性的金催化剂，促进了反应的进行. 


$$
\begin{aligned}
& { }^{t} \mathrm{Bu} \underset{36}{\curvearrowright}+\mathrm{Me}_{3} \mathrm{~N} \rightarrow \mathrm{BH}_{3} \frac{5 \mathrm{~mol} \% \mathrm{Rh}\left(\mathrm{P}^{i} \mathrm{Bu}_{2}{ }^{t} \mathrm{Bu}_{2} \mathrm{BAr}_{\mathrm{F}}\right.}{1,2-\mathrm{F}_{2} \mathrm{C}_{6} \mathrm{H}_{4}} \\
& { }^{t} \mathrm{Bu} \overbrace{37}^{\mathrm{B}_{3}^{\mathrm{H}}}{ }^{\mathrm{H}_{2}} \mathrm{NMe}_{3} \\
& \text { ca. } 70 \% \text { conversion }
\end{aligned}
$$

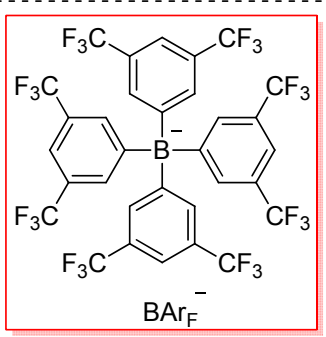

$\left[\mathrm{Rh}\left(\mathrm{P}^{i} \mathrm{Bu}_{2}{ }^{t} \mathrm{Bu}\right)_{2}\right]^{+}$

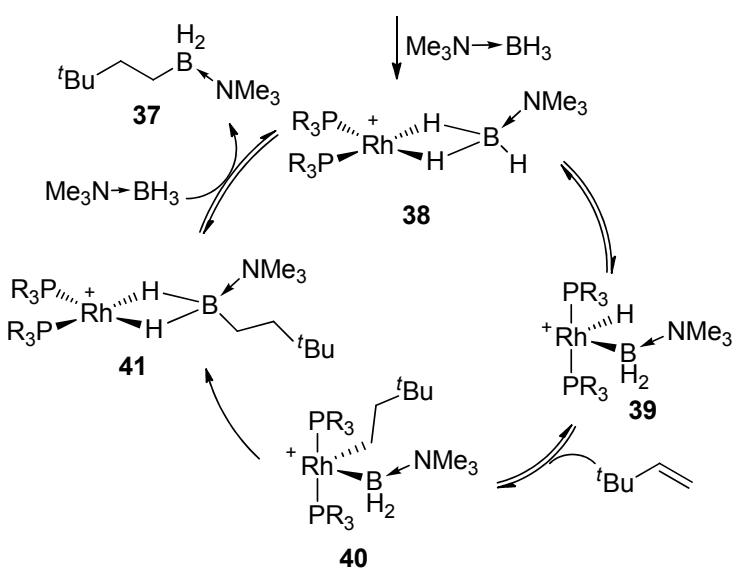

图式 $14 \mathrm{Rh}$ 催化的烯烃和三甲胺嗍烷加合物的硼氢化反应

Scheme 14 Rh-catalyzed hydroboration of alkene with $\mathrm{Me}_{3} \mathrm{~N}$-borane adduct
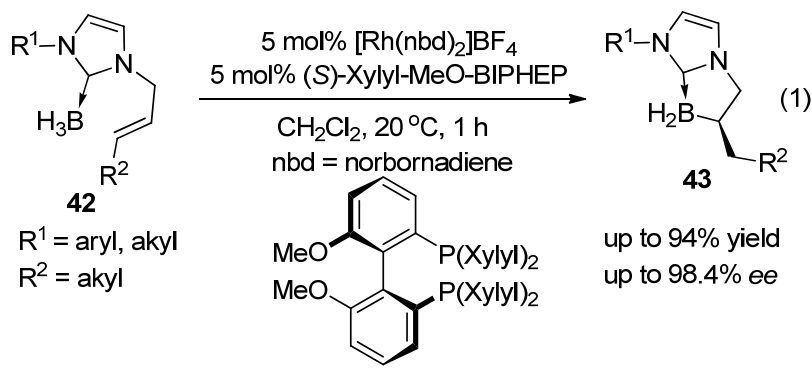

up to $94 \%$ yield up to $98.4 \%$ ee

(S)-Xylyl-MeO-BIPHEP

\section{3 硼烷加合物直接参与的硼氢化反应}

在不添加活化试剂的条件下, 硼烷加合物还可以表 现出亲核性，与活泼炔烃发生硼氢化反应。2014 年, Curran 等 ${ }^{[26]}$ 报道了首例的苯炔的硼氢化反应(Scheme 16). 由于苯炔需要现场制备, 生成苯炔所用的试剂与 常用的硼试剂一般是不兼容的, 这就造成了苯炔嗍氢化 反应难以实现. 而稳定的 $\mathrm{NHC}-\mathrm{BH}_{3}$ 可以在生成苯炔的 环境下稳定存在, 因而可以完成嗍氢化反应. 例如, $\mathrm{NHC}-\mathrm{BH}_{3}$ 可与和苯炔前体 $\mathbf{5 2}$ 在 $\mathrm{KF}$ 和冠醚存在下顺利

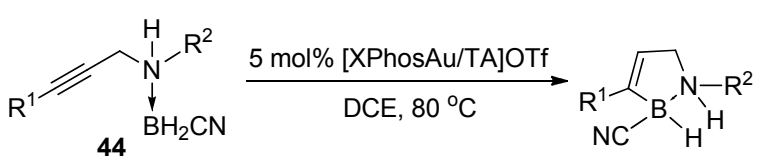

$\mathrm{R}^{1}=$ aryl, alkyl, $\mathrm{H}$ $\mathrm{R}^{2}=\mathrm{H},{ }^{n} \mathrm{Bu}$
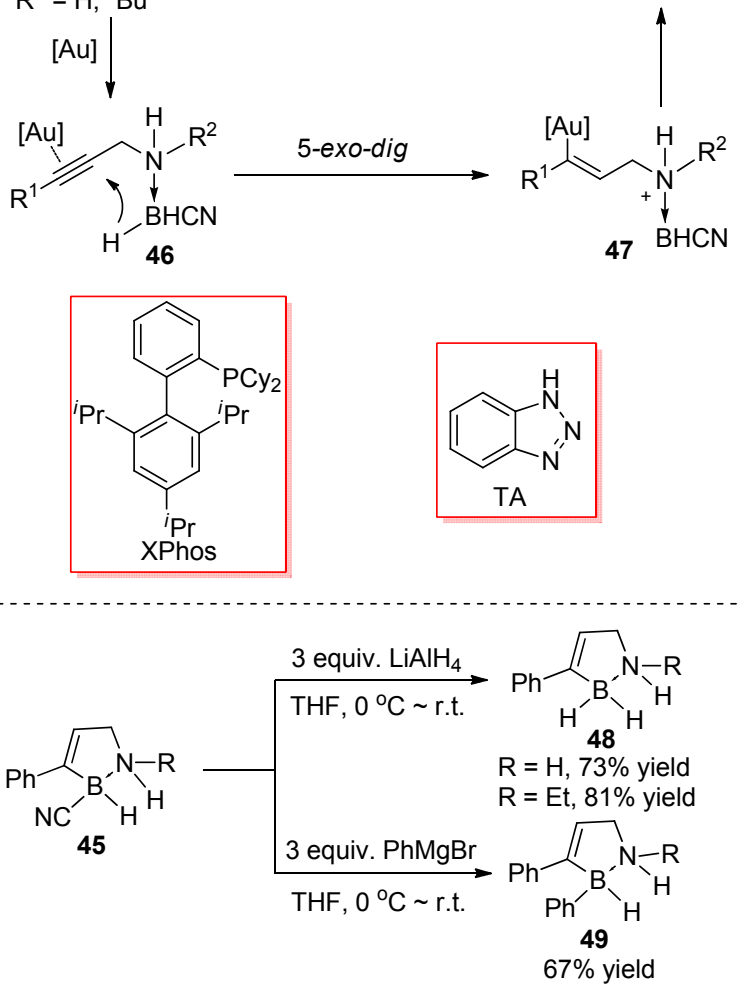

图式 $15 \mathrm{Au}$ 催化的炔烃和硼烷加合物的分子内嗍氢化反应 Scheme 15 Au-catalyzed intramolecular hydroboration of alkynes with borane adducts

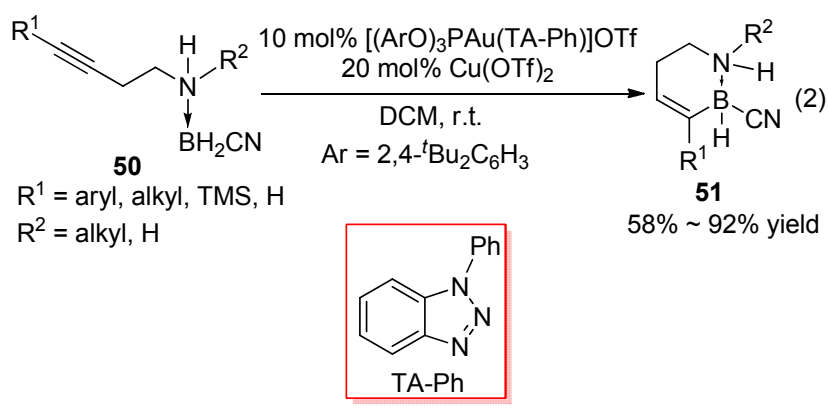

反应生成 NHC 苯基硼烷加合物 53 (Scheme16). 产物 53a 可以与 $\mathrm{I}_{2} 、 \mathrm{Br}_{2} 、 \mathrm{NCS}$ 等反应生成相应的卤代硼烷加 合物 54, 进而水解为苯基硼酸; 也可以与 Selectfluor 作 用生成稳定的 NHC 苯基二氟嗍加合物 $\mathbf{5 5}$, 进而在钯催 化下和溴苯发生交叉偶联反应，以较高的收率生成相应 的联苯化合物 ${ }^{[27]}$.

最近, Curran 等 ${ }^{[28]}$ 报道了 $\mathrm{NHC}-\mathrm{BH}_{3}$ 和乙炔二羧酸 二甲酯的双硼氢化反应，高效合成了 $\mathrm{NHC}$ 稳定的硼杂 环丙烷化合物 56(表 2). 在这个反应中主要的副产物是 1,2-硼氢化反应生成的 $(E)$-烯基硼烷加合物 57. 增大氮 

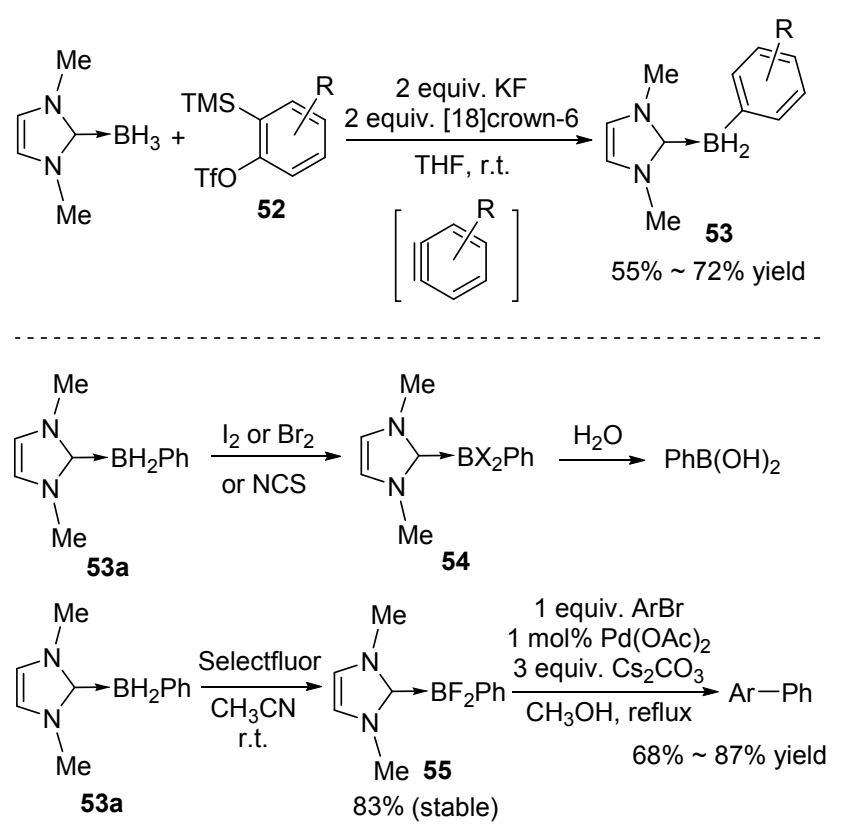

图式 16 苯炔与氮杂环卡宾嗍烷加合物的嗍氢化反应

Scheme 16 Hydroboration of arynes with $\mathrm{NHC}-\mathrm{BH}_{3}$

表 2 由 $\mathrm{NHC}^{-\mathrm{BH}_{3}}$ 和乙炔二羧酸二甲酯合成嗍杂环丙烷化合 物

Table 2 Synthesis of NHC-boriranes by reactions of acetylenedicarboxylate esters with $\mathrm{NHC}-\mathrm{BH}_{3}$

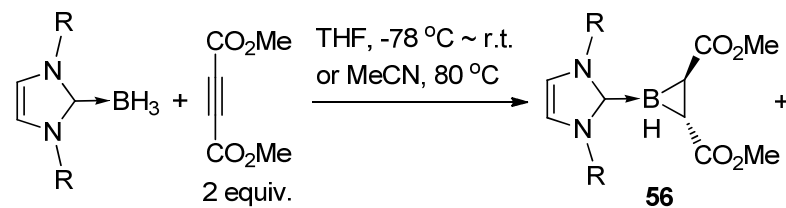<smiles>[R]OC([B]C1N([R])C=CN1[R])=CC(C)OC</smiles>

57

\begin{tabular}{lccc}
\hline \multirow{2}{*}{$\mathrm{R}$} & \multirow{56}{*}{$: \mathbf{5 7}$} & \multicolumn{2}{c}{ Yield $/ \%$} \\
\cline { 3 - 4 } \multicolumn{1}{c}{} & & $\mathbf{5 6}$ & $\mathbf{5 7}$ \\
\hline $\mathrm{Me}$ & $33: 67$ & 19 & 35 \\
${ }^{i} \mathrm{Pr}$ & $52: 48$ & 14 & 29 \\
$2,4,6-\mathrm{Me}_{3} \mathrm{C}_{6} \mathrm{H}_{2}$ & $81: 19$ & 31 & 10 \\
$2,6-{ }^{i} \mathrm{Pr}_{2} \mathrm{C}_{6} \mathrm{H}_{3}$ & $86: 14$ & 80 & 5 \\
\hline
\end{tabular}

杂环卡宾氮上取代基的位阻可以提高硼杂环丙烷产物 的选择性. 化合物 56 和 57 不能互相转化. 通过密度泛 函理论(DFT)计算, 他们给出了可能的反应机理(Scheme

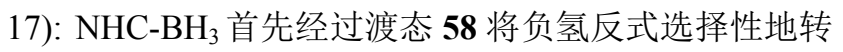
移至炔键生成两性离子中间体 59, 随即溶剂 THF 与砋 配位形成更稳定的四配位的嗍鎓阳离子和乙烯基负离 子的离子对中间体 60 , 随后 $\mathrm{THF}$ 配合的嗍鎓阳离子将 会从烯烃的阴离子 $\pi$ 轨道方向加成, 其对应的过渡态为 61. 通过 Oppenheimer 分子动力学轨道模拟, 作者证明 了由单一过渡态 61 可以同时得到嗍杂环丙烷和烯烃硼

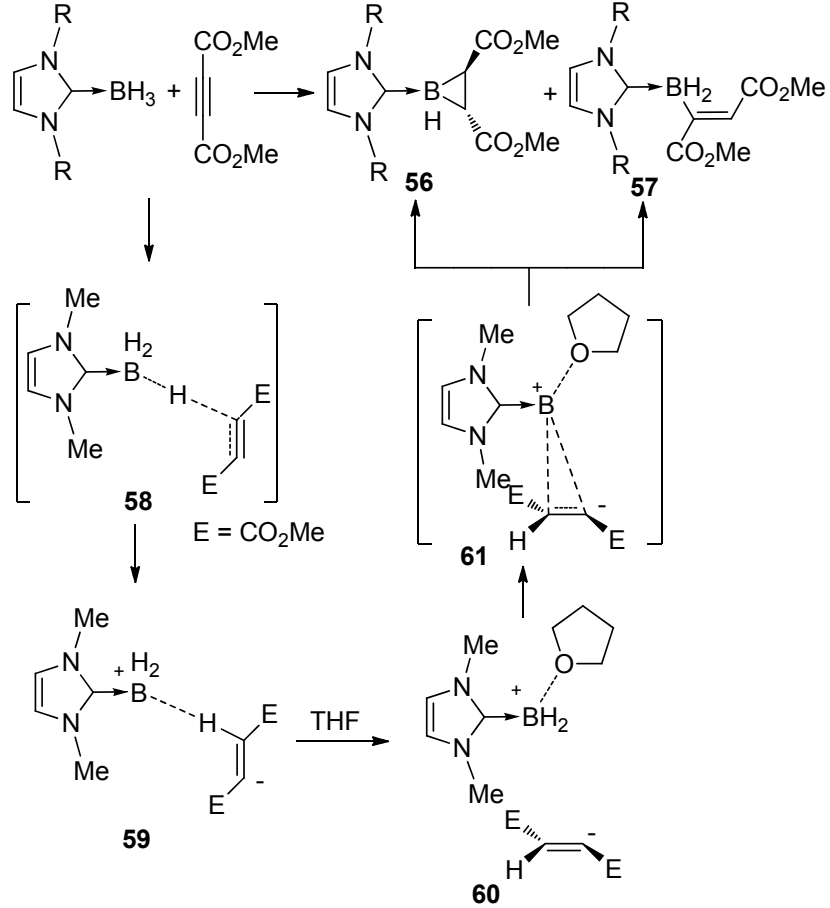

图式 17 合成硼杂环丙烷反应机理

Scheme 17 Mechanism of synthesis of NHC-boriranes

烷两种产物: 硼首先和烯烃的两端分别成键, 生成三元 环中间体，如果发生 1,2-氢迁移则得到嗍杂环丙烷产物 56, 如果发生 $\mathrm{C}-\mathrm{B}$ 键的断裂, 则生成形式上单次嗍氢 化产物 $\mathbf{5 7}$.

\section{$3 \mathrm{C}-\mathrm{H}$ 键嗍化}

通过 $\mathrm{C}-\mathrm{H}$ 键的活化/官能团化反应直接构筑 $\mathrm{C}-\mathrm{B}$ 键是合成有机嗍化物的有效途径, 相关研究引起了人们 的关注, 成为了当前有机合成的研究热点之一 ${ }^{[5 \mathrm{~d}, 5 \mathrm{e}]}$, 硼 烷加合物参与的 $\mathrm{C}-\mathrm{H}$ 键直接硼化反应目前也取得了一 定的进展.

2009 年, Vedejs 等 ${ }^{[29]}$ 报道了 $\left[\mathrm{Ph}_{3} \mathrm{C}\right]\left[\mathrm{B}\left(\mathrm{C}_{6} \mathrm{H}_{5}\right)_{4}\right]$ 促进的 茮胺嗍烷 62 加合物分子内的 $\mathrm{C}-\mathrm{H}$ 键喼化反应, 用 ${ }^{n} \mathrm{Bu}_{4} \mathrm{NBH}_{4}$ 还原淬灭反应后，以中等的收率合成了含嗍 氮的五元杂环化合物 63 (Scheme 18). 他们给出了可能 的反应机理: 强 Lewis 酸三苯基碳正离子作为氢受体与 胺嗍烷加合物作用, 得到具有强亲电性的硼鎓阳离子中 间体 64, 该中间体可与另一分子底物作用形成含有氢 桥键的中间体 65 . 随后 64 或 65 与芳环作用形成 $\pi$ 中间 体 66, 进而生成 Wheland 中间体 67, 经历过渡态 68 脱 去 $\mathrm{H}_{2}$ 得到稳定的化合物 69, 最后被 ${ }^{n} \mathrm{Bu}_{4} \mathrm{NBH}_{4}$ 还原得到 产物 63 .

类似的, Stephan 等 ${ }^{[30]}$ 以 $\left[\mathrm{Ph}_{3} \mathrm{C}\right]\left[\mathrm{B}\left(\mathrm{C}_{6} \mathrm{~F}_{5}\right)_{4}\right]$ 为活化剂 实现了氮杂环卡宾嗍烷加合物的 $\mathrm{C}-\mathrm{H}$ 键㸴化反应 (Scheme 19). 在反应中加入 1 equiv. 的 $\left[\mathrm{Ph}_{3} \mathrm{C}\right]\left[\mathrm{B}\left(\mathrm{C}_{6} \mathrm{~F}_{5}\right)_{4}\right]$, 


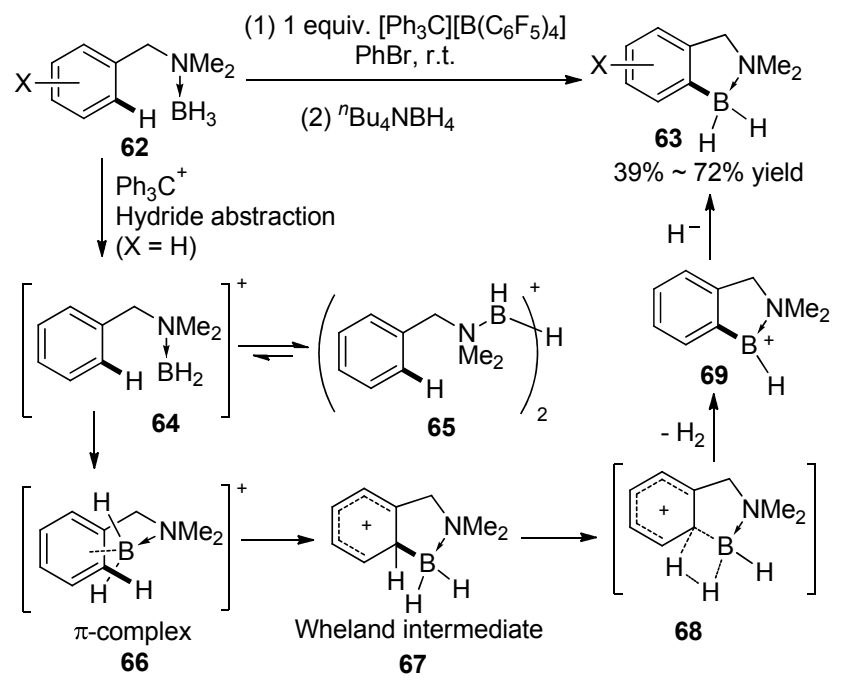

图式 18 苄胺嗍烷加合物的分子内芳基 $\mathrm{C}-\mathrm{H}$ 键嗍化反应 Scheme 18 Intramolecular aromatic $\mathrm{C}-\mathrm{H}$ bond borylation of benzyl amine-boranes

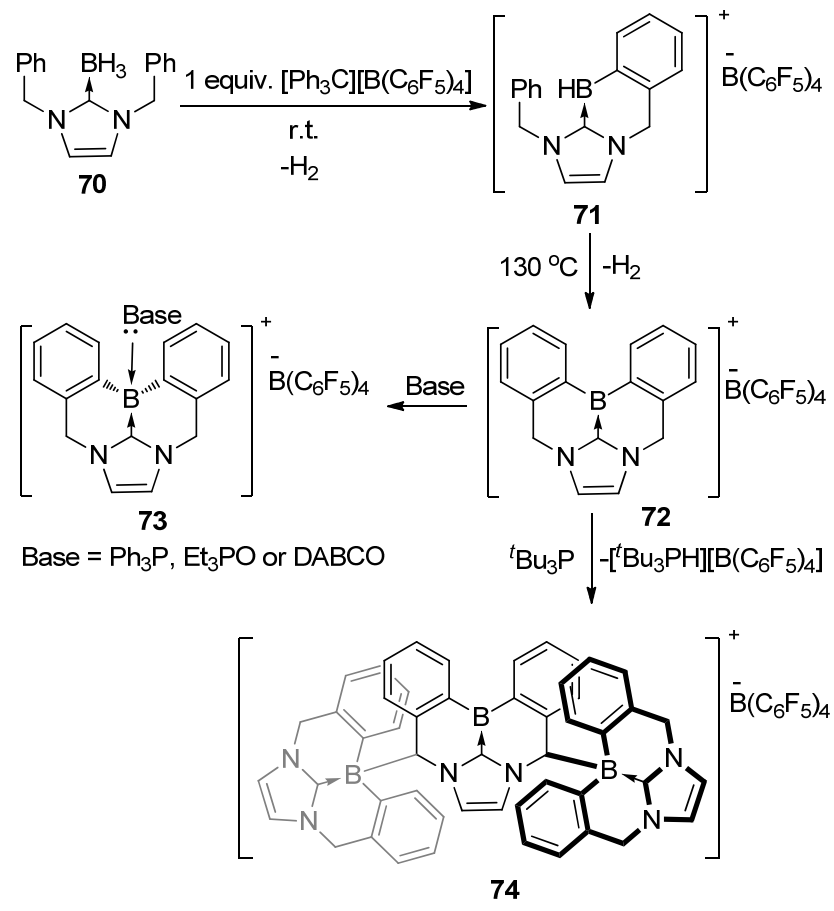

图式 19 氮杂环卡宾嗍烷加合物的 C-H 键嗍化反应

Scheme $19 \mathrm{C}-\mathrm{H}$ bond borylation from NHC-borane

氮杂环卡宾嗍烷加合物 70 可以发生分子内的碳氢硼化 得到产物 71, 在 $130{ }^{\circ} \mathrm{C}$ 的反应温度下, 71 可以继续发生 分子内脱氢硼化生成具有平面结构的 72.72 可以与电子 供体 $\mathrm{PPh}_{3} 、 \mathrm{Et}_{3} \mathrm{PO}$ 或 $\mathrm{DABCO}$ 相互作用形成相应的加合 物 73, 表现出经典的 Lewis 酸碱对的性质. 当加入 ${ }^{t} \mathrm{Bu}_{3} \mathrm{P}$ 时, 三分子的 72 可以发生脱氢偶联形成含有三个硼中 心的正离子化合物 74. 这个方法也为合成平面型的硼 鎓阳离子化合物提供了新的思路.

2011 年, Vedejs 等 ${ }^{[31]}$ 以催化量的 $\mathrm{Tf}_{2} \mathrm{NH}$ 实现了脂肪
胺嗍烷加合物 75 的分子内 $\mathrm{C}-\mathrm{H}$ 键硼化反应，合成了 B-N 杂五元环状化合物 76 (Scheme 20). 此反应需要相 对较高的温度 $\left(>120{ }^{\circ} \mathrm{C}\right)$. 作者推测了反应机理: 强酸 $\mathrm{Tf}_{2} \mathrm{NH}$ 作为氢受体活化嗍烷加合物形成中间体 77, 77 也 是催化循环中的真实催化剂, 其可与另一分子底物形成 强亲电性硼鎓阳离子中间体 $\mathbf{7 8}$, 然后在较高的温度下 离去一分子 $\mathrm{H}_{2}$ 发生碳氢插入反应得到 79 或 80 , 随后与 另一分子底物氢交换生成产物 76, 并且重新生成活性 催化剂 77, 80 也可被还原淬灭得到最终产物 76.

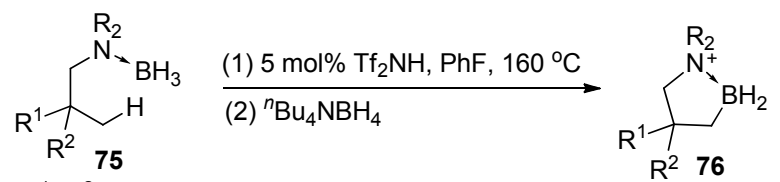

$\mathrm{R}^{1}, \mathrm{R}^{2}=$ aryl, akyl, $\mathrm{H}$ $75 \% \sim 96 \%$ yield

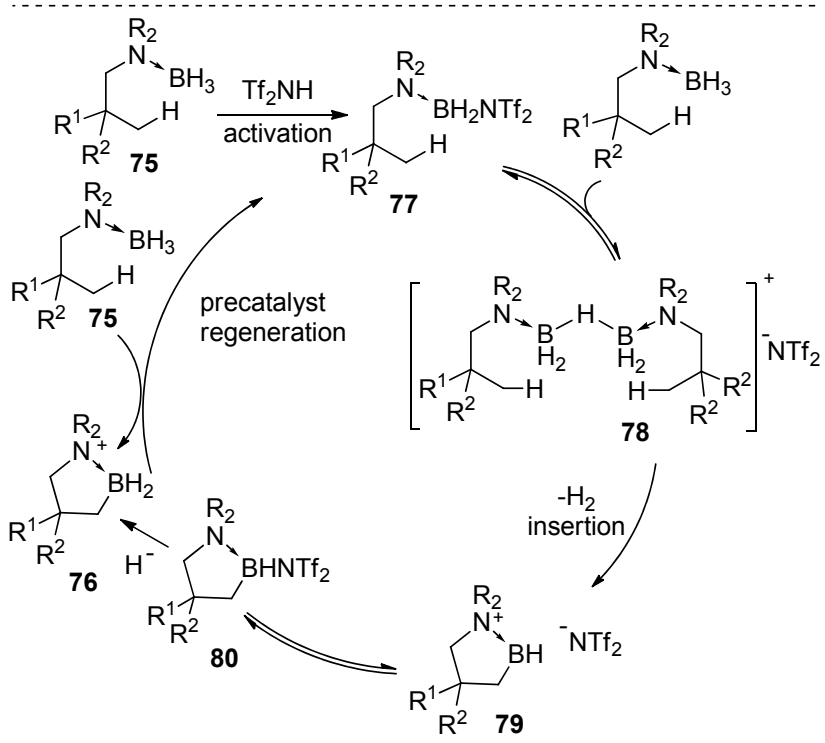

图式 20 脂肪胺嗍烷加合物的分子内 $\mathrm{C}-\mathrm{H}$ 键嗍化反应 Scheme 20 Intramolecular $\mathrm{C}-\mathrm{H}$ bond borylation from benzyl alkyl amine-borane

2013 年, Vedejs 等 ${ }^{[32]}$ 以催化量的 $\mathrm{Tf}_{2} \mathrm{NH}$ 也实现了苄 胺硼烷加合物的分子内芳烃的 $\mathrm{C}-\mathrm{H}$ 键硼化反应(Eq. 3). 与 $\left[\mathrm{Ph}_{3} \mathrm{C}\right]\left[\mathrm{B}\left(\mathrm{C}_{6} \mathrm{H}_{5}\right)_{4}\right]$ 相比, 该反应需要较高的温度, 而反 应的的选择性和收率有较大的提高.

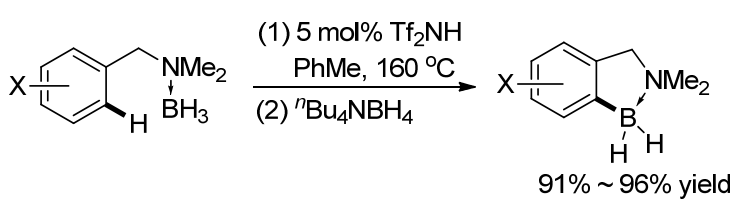

2013 年, Vedejs 等 ${ }^{[33]}$ 报道了 $\mathrm{Tf}_{2} \mathrm{NH}$ 促进的分子内膦 嗍烷加合物 81 的 C- $\mathrm{H}$ 键嗍化反应(Scheme 21). 此反应 的中间体 82 可以被还原淬灭得到五元磷硼杂环化合物 83 , 也可以与 $\mathrm{KHF}_{2}$ 作用得到邻苯酚三氟硼酸钾化合物 84，后者也被用到了S Suzuki 反应中. 


\section{4 卡宾对 $B-H$ 键的插入反应}

金属催化的卡宾对碳-氢键及杂原子-氢键的插入 反应具有反应活性高、选择性可控、条件温和、原子经 济性好等特点，是构筑碳一碳键及碳-杂原子键的一类简 单高效的方法, 在有机合成中有着十分广泛的应用 ${ }^{[34]}$. 硼烷一般表现出 Lewis 酸的性质, 是难以与卡宾进行插 入反应的. 而稳定的嗍烷加合物由于硼的空轨道被一对 电子填充, 显著提高了硼中心电子云密度, 使得 $\mathrm{B}-\mathrm{H}$ 键插入反应得以实现.
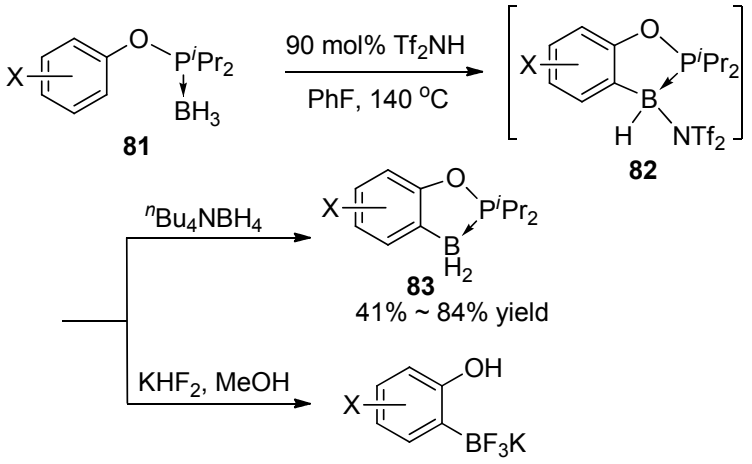

82

$34 \% \sim 94 \%$ yield

图式 $21 \mathrm{Tf}_{2} \mathrm{NH}$ 促进的膦嗍烷加合物分子内的 $\mathrm{C}-\mathrm{H}$ 键嗍化 反应

Scheme $21 \mathrm{Tf}_{2} \mathrm{NH}$-promoted intramolecular $\mathrm{C}-\mathrm{H}$ borylation from phosphine-borane

1993 年, Foucaud 等 ${ }^{[35]}$ 通过氯仿与氢氧化钠产生的 二氯卡宾与嗍烷保护的磷氮杂六元环状化合物作用时, 除了发生环丙烷化反应外，意外地发现二氯卡宾还能对 $\mathrm{B}$ - $\mathrm{H}$ 键发生插入反应(Scheme 22). 随后 Carboni 等 ${ }^{[36]}$ 报道了二氯卡宾对不同的胺和膦磀烷加合物的插入反 应, 除了单次 $\mathrm{B}-\mathrm{H}$ 键的插入产物 87 , 还观察到了二次 插入产物 88 和三次插入产物 89.

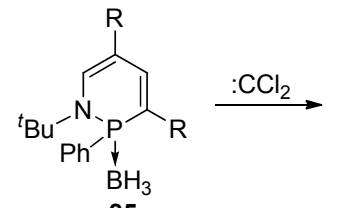

85

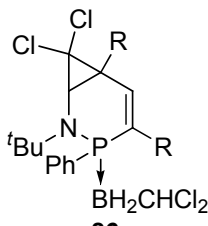

86

$$
\begin{aligned}
& \mathrm{L} \rightarrow \mathrm{BH}_{2} \mathrm{X} \stackrel{: \mathrm{CCl}_{2}}{\longrightarrow} \mathrm{L} \rightarrow \mathrm{BHXCHCl}{ }_{2}+\mathrm{L} \rightarrow \mathrm{BX}\left(\mathrm{CHCl}_{2}\right)_{2}+\mathrm{L} \rightarrow \mathrm{B}\left(\mathrm{CHCl}_{2}\right)_{3} \\
& \mathrm{~L}=\mathrm{R}_{3} \mathrm{~N}, \mathrm{R}_{3} \mathrm{P} \quad 87 \quad 88 \quad 89 \text { (when } \mathrm{X}=\mathrm{H} \text { ) }
\end{aligned}
$$$$
\mathrm{X}=\mathrm{H}, \mathrm{Cl}, \mathrm{Br}, \mathrm{CN}
$$

图式 22 二氯卡宾对 $\mathrm{B}-\mathrm{H}$ 键插入反应

Scheme 22 Insertion reaction of dichlorocarbene into $B-H$ bonds

1996 年, Imamoto 等 ${ }^{[37]}$ 报道了使用现场制备的高活 性甲基或乙基钐卡宾对膦硼烷的 $\mathrm{B}-\mathrm{H}$ 键插入反应. 该 反应具有很大的局限性, 除了要使用大过量的钐卡宾以 外，反应只能向嗍原子上引入甲基或者乙基(Eq. 4).

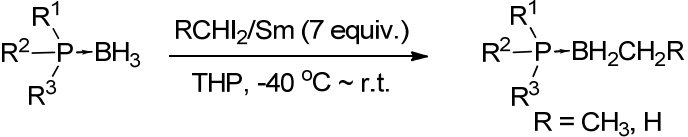

$$
\begin{aligned}
& \mathrm{Cy}_{3} \mathrm{P} \rightarrow \mathrm{BH}_{2} \mathrm{CH}_{3} \quad \mathrm{Cy}_{3} \mathrm{P} \rightarrow \mathrm{BH}_{2} \mathrm{CH}_{2} \mathrm{CH}_{3} \quad{ }^{n} \mathrm{Bu}_{3} \mathrm{P} \rightarrow \mathrm{BH}_{2} \mathrm{CH}_{3} \\
& 72 \% \text { yield } \quad 70 \% \text { yield } \quad 76 \% \text { yield } \\
& { }^{n} \mathrm{Bu}_{3} \mathrm{P} \rightarrow \mathrm{BH}_{2} \mathrm{CH}_{2} \mathrm{CH}_{3} \quad \mathrm{PhMe}_{2} \mathrm{P} \rightarrow \mathrm{BH}_{2} \mathrm{CH}_{3} \\
& 70 \% \text { yield } \quad 68 \% \text { yield } \\
& \mathrm{PhMe}_{2} \mathrm{P} \rightarrow \mathrm{BH}_{2} \mathrm{CH}_{2} \mathrm{CH}_{3} \quad{ }^{t} \mathrm{Bu}_{3} \mathrm{P} \rightarrow \mathrm{BH}_{2} \mathrm{CH}_{3} \\
& 62 \% \text { yield trace }
\end{aligned}
$$

催化卡宾对 $\mathrm{B}$ - $\mathrm{H}$ 键的插入反应直到近几年取得突 破. 2013 年, 朱守非、周其林等 ${ }^{[38]}$ 报道了 $\mathrm{Cu}$ 催化的重氮 酯或重氮酮 90 衍生的卡宾对胺硼烷加合物和膦砋烷加 合物的 $\mathrm{B}-\mathrm{H}$ 键插入反应，在温和条件下以中等或很高 的收率得到相应的 $\alpha$-酯基或 $\alpha$-羰基取代硼烷产物 91 (Eq. 5). 他们还使用铜与手性螺环双啞唑啉配体 94 的络 合物作为催化剂，实现了 $\alpha$-重氮酯 92 对二甲基膦嗍烷 加合物 93 的不对称 $\mathrm{B}-\mathrm{H}$ 键插入反应，获得了很高的对 映选择性(Eq. 6). 重氮底物的酯基部分和硼烷加合物的 Lewis 碱结构对反应的对映选择性影响都很大. B-H 键 插入反应产物 95a 还能以高收率、高度构型保持地转化 为手性硼酸频哪醇酯 97 这一重要的有机硼化合物 (Scheme 23). 当酯基存在时, 95a 不能直接转化为频哪 醇硼酸酯，而是发生脱硣反应，所以酯基需要被还原才 可进行后续转化. 随后工作中, 他们还实现了铜催化 $\alpha$ 重氮酮 98 衍生的卡宾对 $\mathrm{B}-\mathrm{H}$ 键的不对称插入反应 ${ }^{[39]}$,

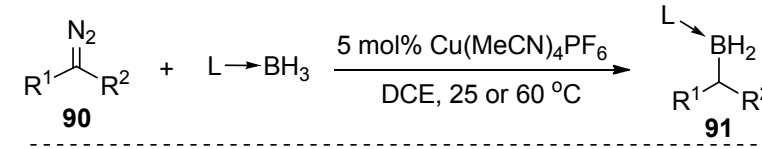

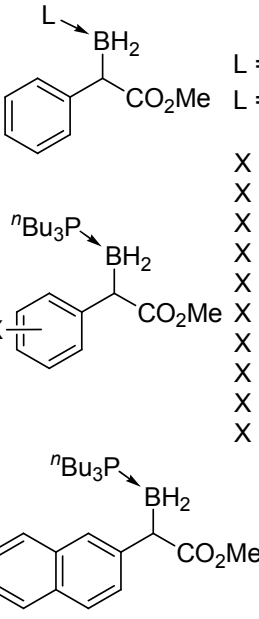

91\% yield

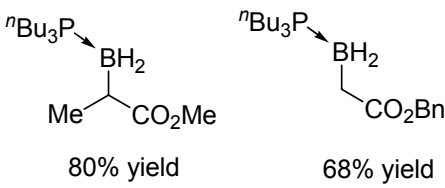

$\mathrm{L}={ }^{n} \mathrm{Bu}_{3} \mathrm{~N}, 83 \%$ yield

$\mathrm{L}=\mathrm{Ph}_{3} \mathrm{P}, 80 \%$ yield

$\mathrm{X}=\mathrm{H} \quad 89 \%$ yield

$\mathrm{X}=2-\mathrm{Cl} \quad 93 \%$ yield

$\mathrm{X}=3-\mathrm{Cl} \quad 86 \%$ yield

$\mathrm{X}=4-\mathrm{Cl} \quad 88 \%$ yield $\mathrm{X}=2-\mathrm{Me} \quad 78 \%$ yield $\begin{array}{ll}X=3-M e & 83 \% \text { yield } \\ X & =43 \% \text { yield }\end{array}$ $\mathrm{X}=2-\mathrm{MeO} 76 \%$ yield $\mathrm{X}=3-\mathrm{MeO} 81 \%$ yield $\mathrm{X}=4-\mathrm{MeO} 82 \%$ yield<smiles>CCCCCC(C(=O)OC)c1ccsc1</smiles>

$66 \%$ yield

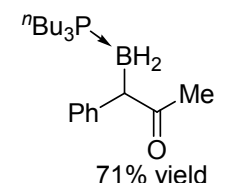




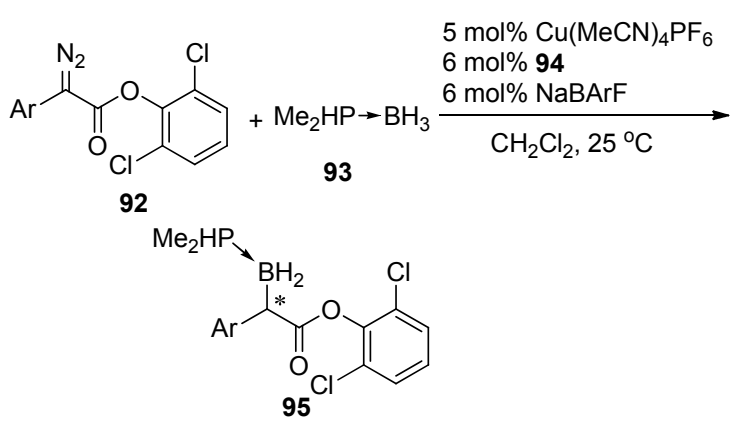

up to $96 \%$ yield up to $94 \%$ ee

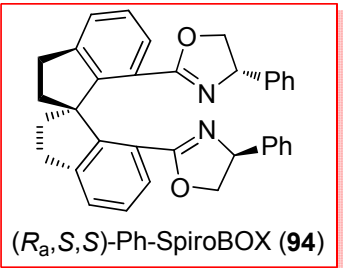

取得了较高的收率和最高达 $83 \%$ ee 的对映选择性(Eq.

7).

2013 年, Curran 等 ${ }^{[40]}$ 报道了 Rh(II)催化的 $\alpha$-重氮羰

(6)

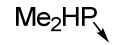

$\mathrm{Me}_{2} \mathrm{HP}$

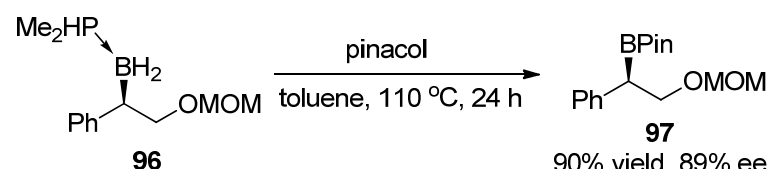
$91 \%$ yield, $91 \%$ ee $90 \%$ yield, $89 \%$ ee

图式 $23 \mathrm{~B}-\mathrm{H}$ 键插入产物转化

Scheme 23 Transformation of $\mathrm{B}-\mathrm{H}$ bond insertion product 基化合物 100 衍生的卡宾和氮杂环卡宾嗍烷加合物 101 的插入反应，以中等或较高的收率得到了一系列的 $\alpha$-硼 烷羰基化合物 102 (Eq. 8). 随后的工作中他们也实现了 $\mathrm{Rh}$ (II) 催化的 $\alpha$-重氮酯 103 衍生的卡宾对膦和胺等硼烷 加合物的插入反应，同样获得了中等或较高的收率(Eq. 9) ${ }^{[41]}$.

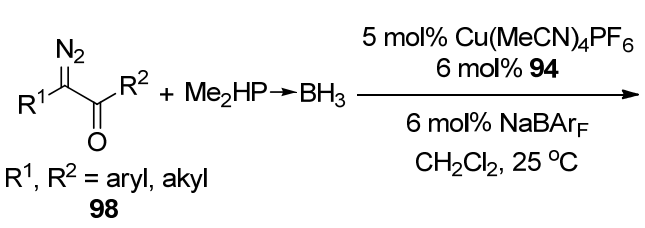

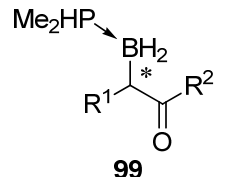

99

up to $86 \%$ yield up to $83 \%$ ee<smiles>CCCCC(=O)C[B]C1N(C)C=CN1C</smiles>

$58 \%$ yield<smiles></smiles>

$74 \%$ yield<smiles>CCC(=O)C[B]C1N(C)C=CN1C</smiles><smiles>COC(=O)C(Br)C(=O)OC</smiles><smiles>CN(C)C(=O)C[BH2-]C1N(C)C=CN1C</smiles>

$55 \%$ yield<smiles>CCOC(=O)C[B]C1N(C)C=NN1C</smiles>

$52 \%$ yield<smiles>COC(=O)C([B]C1N(C)C=CN1C(C)=O)C(C)=O</smiles>

$73 \%$ yield<smiles>CCOC(=O)C[B]C1N(C)c2ccccc2N1C</smiles> 


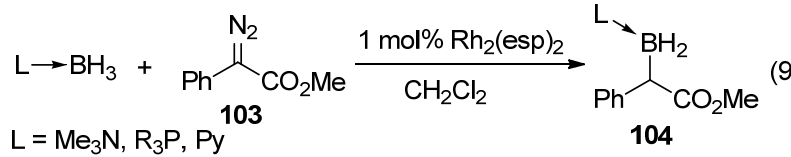

$$
\begin{aligned}
& 30 \% \sim 79 \% \text { yield }
\end{aligned}
$$

2015 年, 徐明华等 ${ }^{[42]}$ 报道了 $\mathrm{Rh}(\mathrm{I})$ 催化的 $\alpha$-重氮酯 和 $\alpha$-重氮酮卡宾对胺嗍烷加合物的不对称 $\mathrm{B}-\mathrm{H}$ 键插入 反应. 他们使用 $C_{1}$ 对称性的手性二环 [2.2.2]辛二烯配体 105 与 $\mathrm{Rh}(\mathrm{I})$ 的络合物作为催化剂, 顺利实现了上述转 化, 以较高的收率和优异的对映选择性合成了手性 $\alpha$-硼 烷羰基化合物 106 和 107 (Scheme 24).

最近, Gouverneur 等 ${ }^{[43]}$ 报道了 $\mathrm{Cu}(\mathrm{I})$ 催化的 $\alpha$-三氟甲 基重氮化合物 108 衍生的卡宾对 $\mathrm{B}-\mathrm{H}$ 键的不对称插入 反应，合成了手性 $\alpha$-三氟甲基有机嗍化合物 110 (Eq. $10)$. 当以手性双二噁唑啉 109 为配体时, 可以得到最高 $81 \% e e$ 的对映选择性.
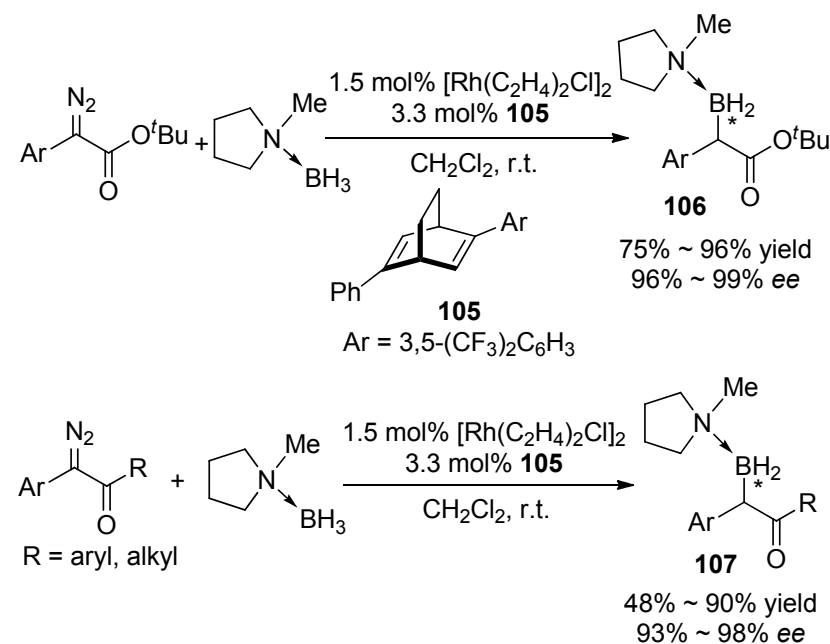

图式 $24 \mathrm{Rh}(\mathrm{I})$ 催化的卡宾对胺硼烷加合物的不对称 B-H 键 插入反应

Scheme 24 Rhodium(I)-catalyzed asymmetric B-H bond insertion of amine-borane adducts

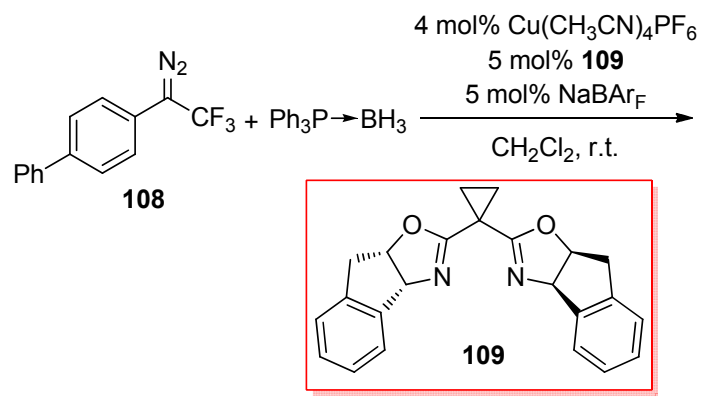

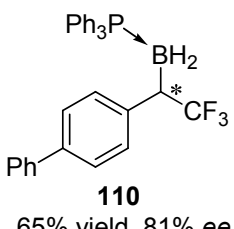

(10)
反应可以高效地构筑 $\mathrm{C}-\mathrm{B}$ 键, 为有机嗍化合物的合成 提供了一个新的有效方法，逐渐受到重视. 但是，使用 重氮化合物作为卡宾前体进行 $\mathrm{B}-\mathrm{H}$ 键插入反应也存在 着明显的局限性. 一方面，重氮化合物通常具有较强的 毒性和爆炸性，限制了上述反应的实际应用．另一方面， 重氮化合物通常需要一个拉电子基团存在才能稳定，这 就限制了产物的类型. 最近，朱守非、周其林等 ${ }^{[44]}$ 在催 化 $\mathrm{B}-\mathrm{H}$ 键插入反应研究上取得了重要进展, 部分解决 了上述问题. 他们以共轭的炔烃 111 为卡宾前体, 在铜 或铑金属络合物催化下实现了 $\mathrm{B}-\mathrm{H}$ 键插入反应，为有 机喼化合物的合成提供了新的方法(Eq. 11). 该反应条 件温和，对于羰基烯炔和亚胺烯炔两类卡宾前体和胺基 硼烷加合物的反应都能够给出近乎定量的收率. 当使用 手性双铑络合物催化剂时，还能实现反应的不对称转 化，给出高达 $96 \%$ ee 的对映选择性(Eq. 12). 反应产物 112a 可以方便地转化为在有机合成中广泛应用的双芳 基甲醇 113、硣酸酯 114 和 115 等重要合成砌块(Scheme 25). 动力学研究表明, 该反应对催化剂和炔烃底物是 1 级反应, 对硼烷加合物是 0 级反应, $\mathrm{B}-\mathrm{H}$ 键插入过程不 是决速步. DFT 计算表明金属卡宾的产生是决速步, B一 $\mathrm{H}$ 键插入经历了协同的机理, 通过三元环过渡态完成的 (Scheme 26).

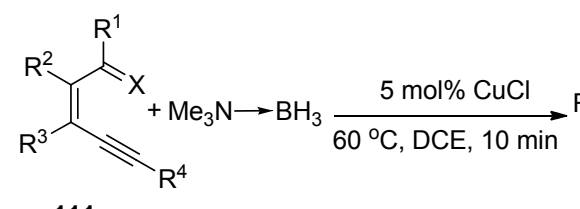<smiles>[R]c1[X]c(C([R])[B]NC)c([R])c1[R]</smiles>

112

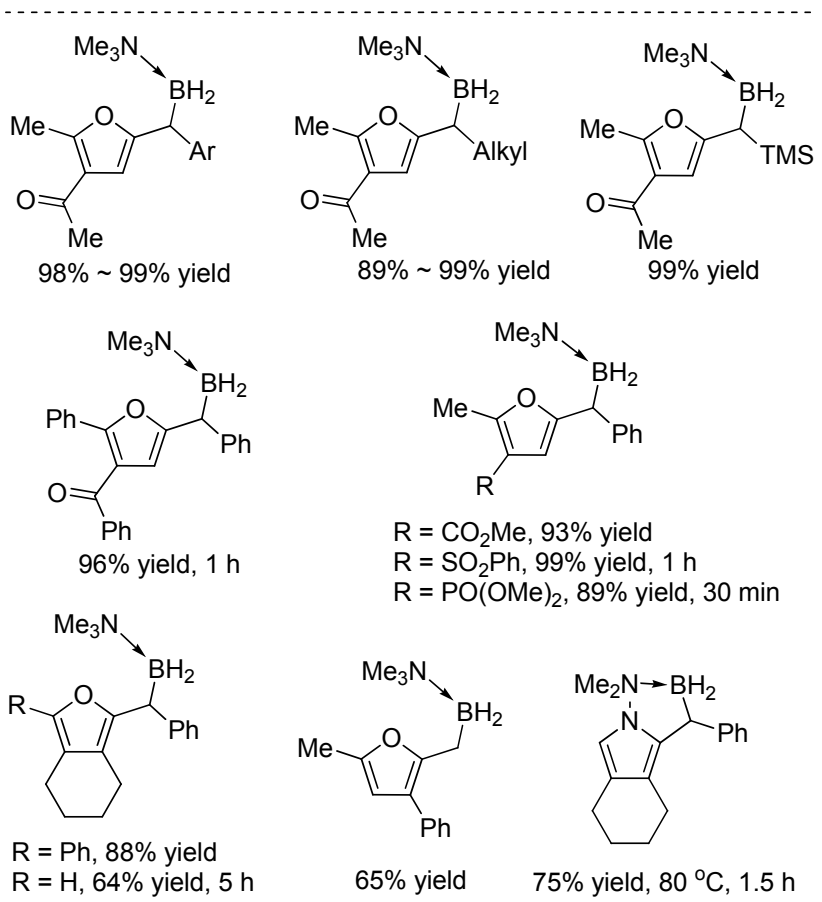



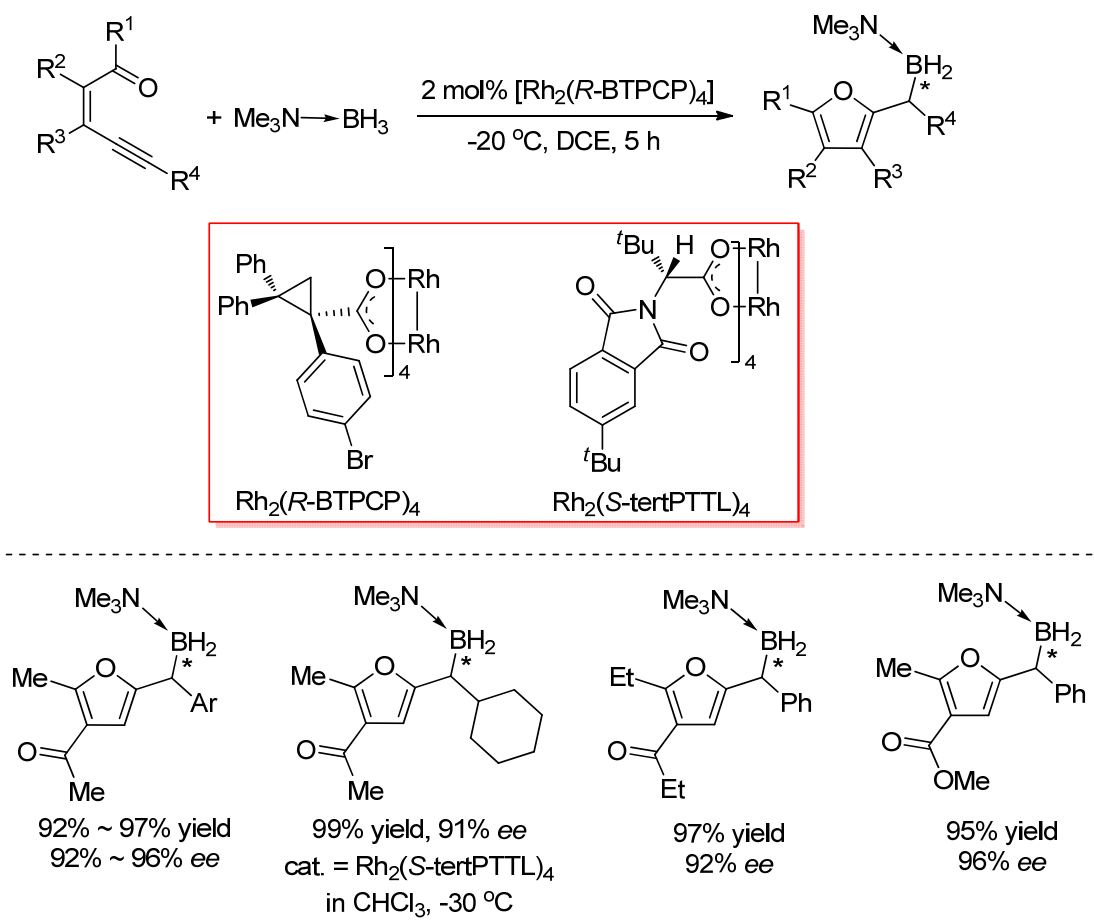

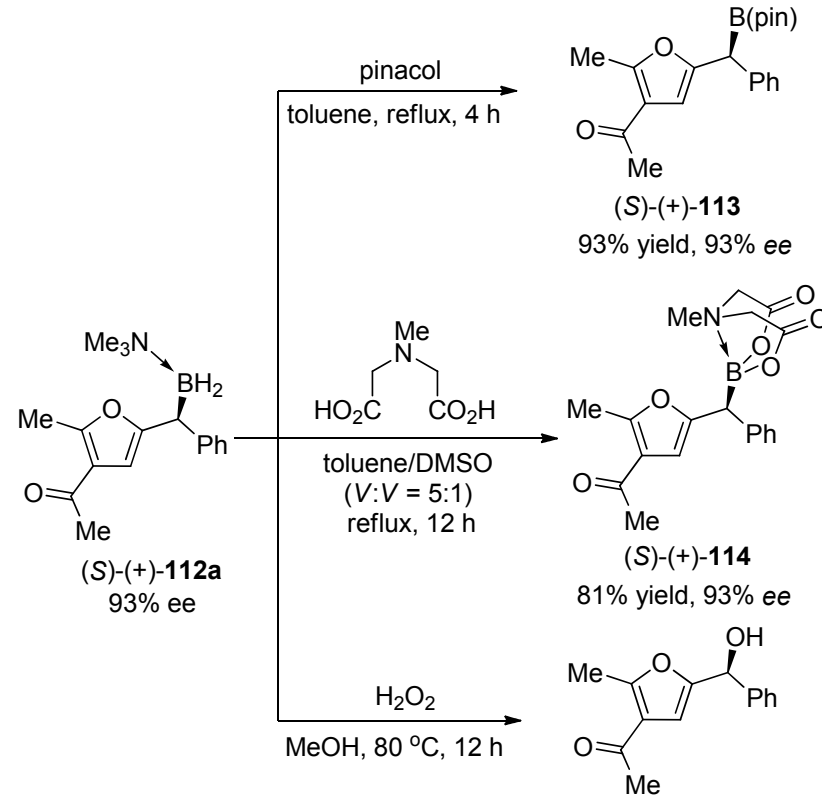

$(S)-(+)-115$

$83 \%$ yield, $93 \%$ ee

图式 25 炔烃为卡宾前体的 $\mathrm{B}-\mathrm{H}$ 键插入产物转化

Scheme 25 Transformations of $\mathrm{B}-\mathrm{H}$ bond insertion product using alkynes as carbene precursors

\section{5 硼自由基参与的串联环化反应}

㸴的自由基化学一直备受关注 ${ }^{[45]}$. 近些年来, 硓烷 加合物的自由基中间体也逐渐得到认识 ${ }^{[46]}$, 并且用在 了合成领域, 如: 黄原酸酯化合物脱氧反应 ${ }^{[47]}$ 、有机卤 化物的脱卤反应 ${ }^{[48]}$ 、缺电子烯烃的自由基聚合反应 ${ }^{[49]}$ 、 二硫化合物的均裂取代反应 ${ }^{[50]}$. 最近, 稳定硼烷加合物
的自由基中间体参与的有机嗍化物的合成也取得了一 些重要进展. 2017 年, Curran 和 Taniguchi 等 ${ }^{[51]}$ 报道了氮 杂环卡宾硼烷自由基参与的二炔化合物 118 的串联环化 反应, 得到了多环结构的含硼化合物 119 (Scheme 27). 他们认为反应机理为: 氮杂环卡宾嗍烷加合物在 TBHN 的引发下生成嗍自由基中间体 $\mathbf{1 2 0}$, 随后对二炔底物自 由基加成生成中间体 121, 接着发生自由基环化反应生 成自由基中间体 122, 122 可以与另一分子嗍烷加合物发 生篗氢反应生成产物 $\mathbf{1 1 9}$, 同时再生 $\mathbf{1 2 0}$.

最近，汪义丰等 ${ }^{[52]}$ 报道了氮杂环卡宾硼烷自由基 参与的 1,6-烯炔化合物 123 的环化反应, 得到了硼基团 取代的六元环状化合物 124 和 125 (Scheme 28). 两种产 物的选择性主要受炔和烯烃上的取代基控制的. 他们给 出了可能的反应机理: 氮杂环卡宾嗍烷加合物和自由基 引发剂反应生成硼自由基中间体 120 , 当底物是 $123 \mathrm{a}$ 时, 120 进攻苯基取代的双键生成中间体 126, 随后发生 对炔基的自由基进攻经 6-exo-dig 环化高对映选择性地 得到 127, 最后篗取硫醇上的氢原子生成目标产物 124a, 生成的硫醇自由基可以继续㩲取氮杂环卡宾硣烷加合 物上的氢重新生成 120 . 当底物为 $123 b$ 时, 120 首先进攻 苯基取代的炔键, 随后经关环、氢转移得到目标产物 $125 b$.

\section{6 取代反应}

NHC-嗍烷加合物在碘或者 HOTf 的活化下生成活 性较高的离子型硼烷加合物, 进而和亲核试剂发生取代 反应. 当使用氰基化合物作为亲核试剂时，能够生成 


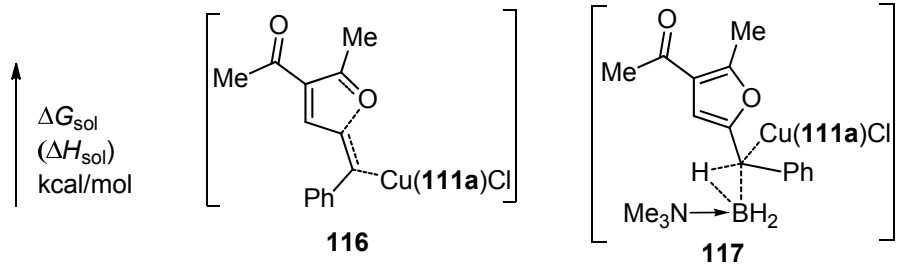

225 $(23.2)$

20.4
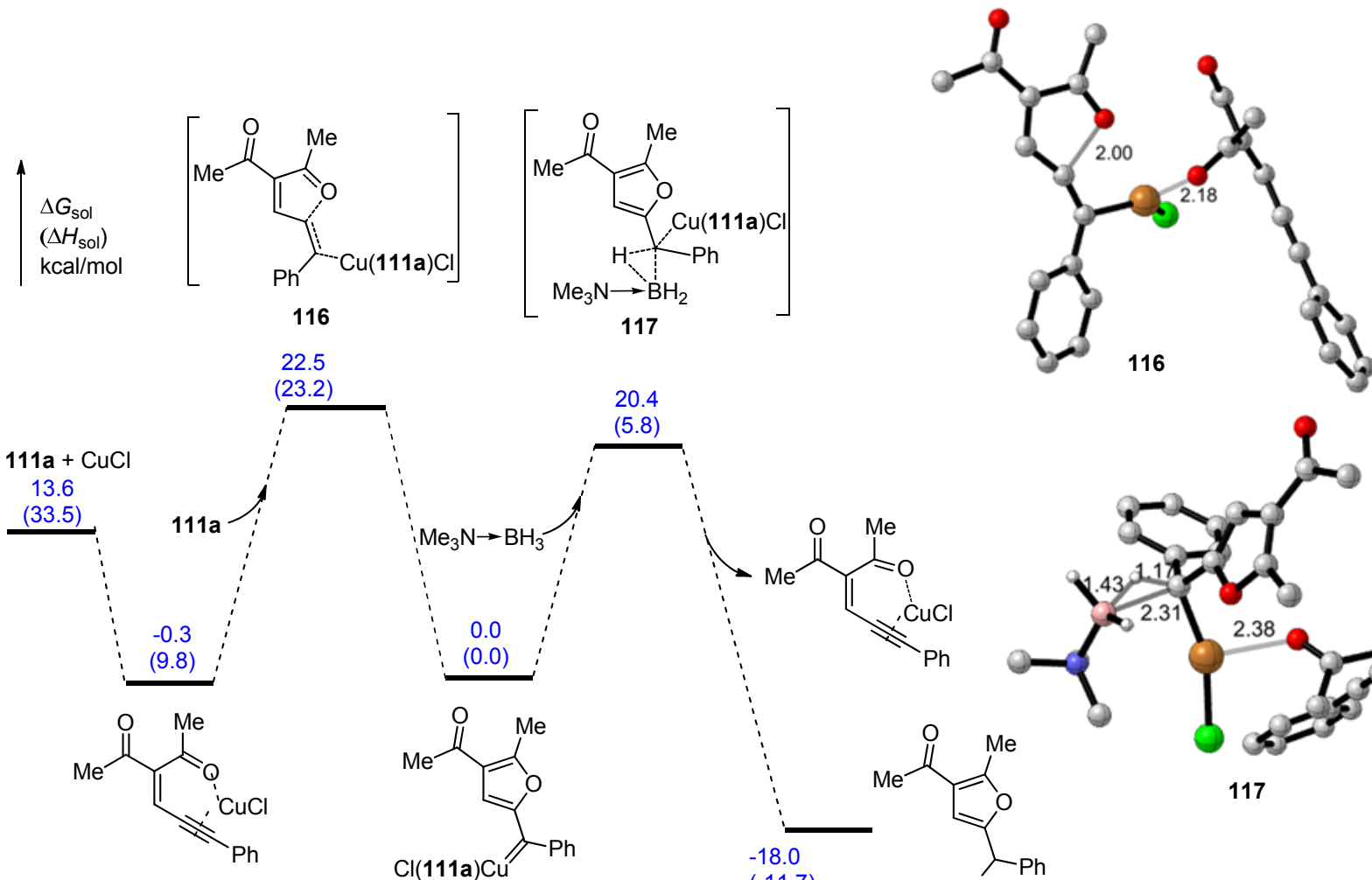

$(5.8)$

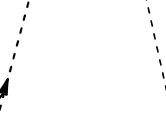

Generation of carbene

图式 $26 \mathrm{CuCl}$ 催化的炔烃为卡宾前体的 $\mathrm{B}-\mathrm{H}$ 键插入反应吉布斯自由能曲线图

Scheme 26 Free energy profile for $\mathrm{CuCl}$-catalyzed $\mathrm{B}-\mathrm{H}$ bond insertion using alkynes as carbene precursors

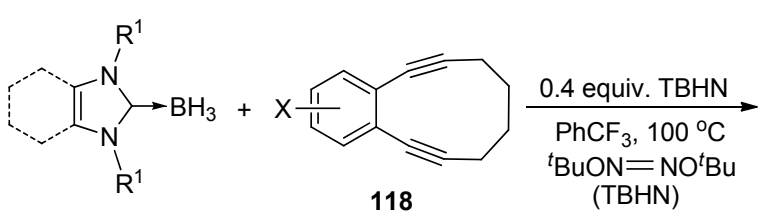<smiles></smiles>

119

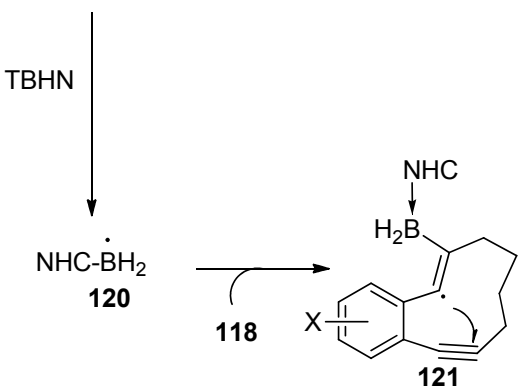

图式 27 嗍自由基参与的二炔环化反应

Scheme 27 Radical boryaltion/cyclization of diynes

C-B 键, 经水解生成嗍取代酰胺 (Scheme 29) ${ }^{[53]}$. NHC碘硼烷加合物在还原剂联苯基锂存在下能够生成硼负 离子, 进而和多种亲电试剂发生取代反应, 构筑 $\mathrm{C}-\mathrm{B}$ 键(表 3) ${ }^{[19]}$. NHC-叠氮嗍烷可以和炔烃发生 $[3+2]$ 环加 成生成硼取代的三氮唑 ${ }^{[54]}$, 由于没有涉及 $\mathrm{C}-\mathrm{B}$ 键的形 成，在此不予展开，感兴趣的读者可以参考相应文献.

\section{7 结论与展望}

有机嗍化合物的合成一直是有机合成中的研究热 点, 在近十年来稳定嗍烷加合物作为一种硼试剂在有机 硼化合物的合成中受到越来越多的关注, 并且表现出了 独特的性质。它们大多易于制备、稳定性高、对水和空 气都不敏感, 以至于常常表现出异于经典 “嗍烷化学” 


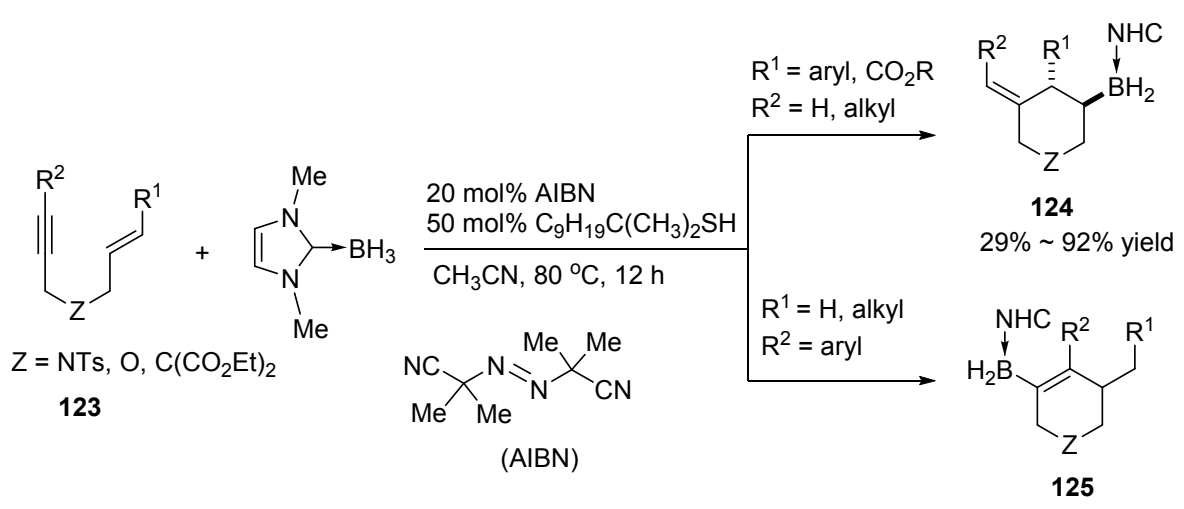

$35 \% \sim 81 \%$ yield

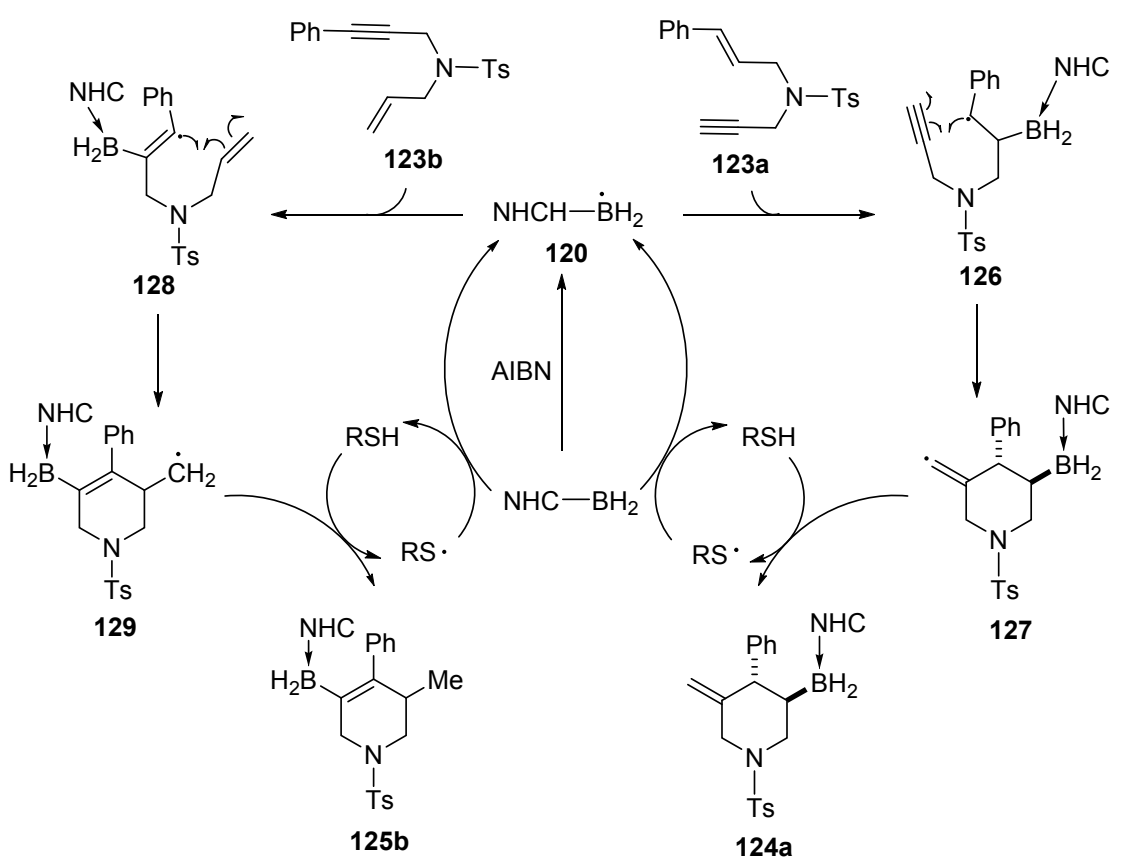

图式 28 嗍自由基参与的 1,6-烯炔环化反应

Scheme 28 Radical boryaltion/cyclization of 1,6-enynes

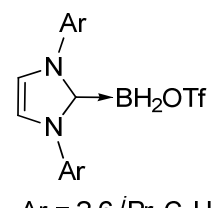

$\mathrm{Ar}=2,6-{ }^{i} \mathrm{Pr}_{2} \mathrm{C}_{6} \mathrm{H}_{3}$
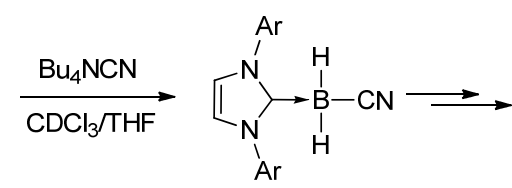

$81 \%$ yield

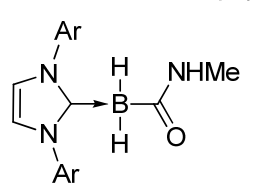

$61 \%$ yield

图式 29 离子型 NHC-硼烷加合物的亲核取代 Scheme 29 Nucleophilic substitution of ionic NHC-borane adducts

的性质. 稳定厥烷加合物作为硼试剂参与的有机嗍化合 物的合成反应不断取得突破, 新的合成方法不断被开发
出来. 稳定硼烷加合物独特的化学性质也为 $\mathrm{C}-\mathrm{B}$ 键形 成反应提供了新的想象空间，而且由其生成的有机嗍化 合物也逐渐得到应用.

但是还应看到，稳定硼烷加合物为硼试剂参与的有 机嗍化物合成反应类型较少, 大多是在试剂量活化剂的 条件下进行反应，金属催化的反应类型较少，反应选择 性控制还是一个挑战性课题，大多数稳定硼烷加合物参 与的 $\mathrm{C}-\mathrm{B}$ 成键反应的机理研究有待进一步深入. 另一 方面, 相关反应生成的有机硼烷加合物的转化方法也比 较少，在合成中的应用有待进一步发掘. 上述问题随着 研究的深入有希望得到解决, 因此, 我们有理由相信未 来稳定硓烷加合物参与的有机嗍化物合成反应及其产 物的应用将会持续受到关注, 也必将为有机硼化学带来 新的机遇. 
表 3 NHC-嗍烷加合物与亲电试剂的取代反应

Table 3 Reactions of electrophiles with NHC-borane adducts

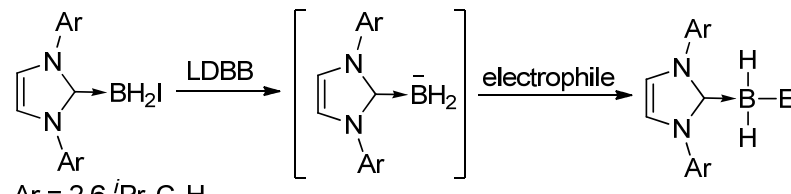

$\mathrm{Ar}=2,6-{ }^{i} \mathrm{Pr}_{2} \mathrm{C}_{6} \mathrm{H}_{3}$

LDBB = lithium di-tert-butylbiphenylide

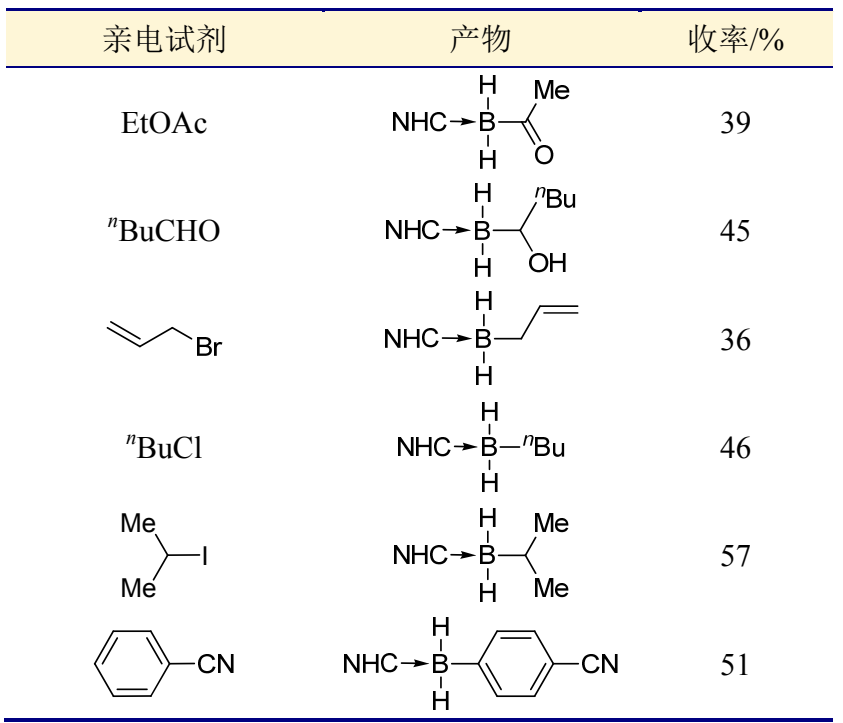

\section{References}

[1] (a) Carboni, B.; Monnier, L. Tetrahedron 1999, 55, 1197.

(b) Staubitz, A.; Robertson, A. P. M.; Sloan, M. E.; Manners, I. Chem. Rev. 2010, 110, 4023.

(c) Staubitz, A.; Robertson, A. P. M.; Manners, I. Chem. Rev. 2010, $110,4079$.

[2] For a recent review, see: (a) Yang, X.; Xie, Z.; He, J.; Yu, L. Chin J. Org. Chem. 2015, 35, 603 (in Chinese).

(阳香华, 谢珍茗, 何军, 余林, 有机化学, 2015, 35, 603.)

For selected examples, see: (b) Blaquiere, N.; Diallo-Garcia, S.; Gorelsky, S. I.; Black, D. A.; Fagnou, K. J. Am. Chem. Soc. 2008, 130, 14034.

(c) Shao, Z.; Fu, S.; Wei, M.; Zhou, S.; Liu, Q. Angew. Chem., Int. Ed. 2016, 55, 14653 .

(d) Zhou, Q.; Zhang, L.; Meng, W.; Feng, X.; Yang, J.; Du, H. Org. Lett. 2016, 18, 5189.

(e) Li, S.; Meng, L.; Du, H. Org. Lett. 2017, 19, 2604.

[3] Curran, D. P.; Solovyev, A.; Brahmi, M. M.; Fensterbank, L.; Malacria, M.; Lacôte, E. Angew. Chem., Int. Ed. 2011, 50, 10294.

[4] For selected reviews, see: (a) Ramachandran, P. V., Brown, H. C. Organoboranes for Syntheses, ACS Symposium Series 783, American Chemical Society, Washington, DC, 2001.

(b) Miyaura, N.; Suzuki, A. Chem. Rev. 1995, 95, 2457.

(c) Braunschweig, H.; Dewhurst, R. D.; Schneider, A. Chem. Rev. 2010, 110, 3924.

(d) Jäkle, F. Chem. Rev. 2010, 110, 3985.

(e) Dembitsky, V. M.; Quntar, A. A. A. A.; Srebnik, M. Chem. Rev. 2011, 111, 209.

(f) Jana, R.; Pathak, T. P.; Sigman, M. S. Chem. Rev. 2011, 111, 1417.

(g) Leonori, D.; Aggarwal, V. K. Angew. Chem., Int. Ed. 2015, 54, 1082 .

[5] For reviews, see: (a) Burgess, K.; Ohlmeyer, M. J. Chem. Rev. 1991, 91, 1179.

(b) Beletskaya, I.; Moberg, C. Chem. Rev. 2006, 106, 2320.

(c) Mkhalid, I. A. I.; Barnard, J. H.; Marder, T. B.; Murphy, J. M.;
Hartwig, J. F. Chem. Rev. 2010, 110, 890

(d) Hartwig, J. F. Chem. Soc. Rev. 2011, 40, 1992.

(e) Ros, A.; Fernández, R.; Lassaletta, J. M. Chem. Soc. Rev. 2014, $43,3229$.

[6] (a) Welch, C. N.; Shore, G. S. Inorg. Chem. 1968, 7, 225. (b) Brown, H. C.; Gupta, S. K. J. Am. Chem. Soc. 1975, 97, 5249.

[7] Zaidlewicz, M.; Brown, H. C.; Siebert, W. Advances in Boron Chemistry, The Royal Society of Chemistry, Cambridge, 1997, 171.

[8] (a) Schaeffer, G. W.; Anderson, E. R. J. Am. Chem. Soc. 1949, 71 , 2143 .

(b) Nainan, K. C.; Ryschkewitsch, G. E. Inorg. Chem. 1969, 8, 2671 .

[9] (a) Baldwin, A. R.; Washburn, R. M. J. Org. Chem. 1961, 26, 3549. (b) Brahmi, M. M.; Monot, J.; Murr, M, D.; Curran, D. P.; Fensterbank, L.; Lacôte, E.; Malacria, M. J. Org. Chem. 2010, 75, 6983.

[10] (a) Brown, H. C.; Chandrasekharan, J. J. Am. Chem. Soc. 1984, $106,1863$.

(b) Kanth, J. V. B. Aldrichim. Acta 2002, 35, 57.

[11] Scheideman, M.; Shapland, P.; Vedejs, E. J. Am. Chem. Soc. 2003, 125, 10502 .

[12] Beak, P. Acc. Chem. Res. 1992, 25, 215.

[13] Clay, J. M.; Vedejs, E. J. Am. Chem. Soc. 2005, 127, 5766.

[14] (a) Shapland, P.; Vedejs, E. J. Org. Chem. 2006, 71, 6666. (b) Karatjas, A. G.; Vedejs, E. J. Org. Chem. 2008, 73, 9508.

(c) Scheideman, M.; Wang, G.; Vedejs, E. J. Am. Chem. Soc. 2008 , 130,8669 .

[15] Pronin, S. V.; Tabor, M. G.; Jansen, D. J.; Shenvi, R. A. J. Am. Chem. Soc. 2012, 134, 2012.

[16] Tabor, M. G.; Shenvi, R. A. Org. Lett. 2015, 17, 5776.

[17] Prokofjevs, A.; Boussonnière, A.; Li, L.; Bonin, H.; Lacôte, E.; Curran, D. P.; Vedejs, E. J. Am. Chem. Soc. 2012, 134, 12281.

[18] Pan, X.; Boussonnière, A.; Curran, D. P. J. Am. Chem. Soc. 2013, 135, 14433.

[19] Monot, J.; Solovyev, A.; Bonin-Dubarle, H.; Derat, É.; Curran, D. P.; Robert, M.; Fensterbank, L.; Malacria, M.; Lacôte, E. Angew Chem., Int. Ed. 2010, 49, 9166.

[20] Boussonnière, A.; Pan, X.; Geib, S. J.; Curran, D. P. Organometallics 2013, 32, 7445.

[21] Sewell, L. J.; Chaplin, A. B.; Weller, A. S. Dalton Trans. 2011, 40, 7499.

[22] Johnson, H. C.; Torry-Harris, R.; Ortega, L.; Theron, R.; McIndoe, J. S.; Weller, A. S. Catal. Sci. Technol. 2014, 4, 3486.

[23] Toure, M.; Chuzel, O.; Parrain, J. L. J. Am. Chem. Soc. 2012, 134, 17892.

[24] Wang, Q.; Motika, S. E.; Akhmedov, N. G.; Petersen, J. L.; Shi, X. Angew. Chem., Int. Ed. 2014, 53, 5418.

[25] Motika, S. E.; Wang, Q.; Akhmedov, N. G.; Wojtas, L.; Shi, X. Angew. Chem., Int. Ed. 2016, 55, 11582.

[26] Taniguchi, T.; Curran, D. P. Angew. Chem., Int. Ed. 2014, 53, 13150.

[27] Nerkar, S.; Curran, D. P. Org. Lett. 2015, 17, 3394.

[28] McFadden, T. R.; Fang, C.; Geib, S. J.; Merling, E.; Liu, P.; Curran D. P. J. Am. Chem. Soc. 2017, 139, 1726.

[29] De Vries, T. S.; Prokofjevs, A.; Harvey, J. N.; Vedejs, E. J. Am. Chem. Soc. 2009, 131, 14679.

[30] Farrell, J. M.; Stephan, D. W. Angew. Chem., Int. Ed. 2015, 54, 5214.

[31] Prokofjevs, A.; Vedejs, E. J. Am. Chem. Soc. 2011, 133, 20056.

[32] Prokofjevs, A.; Jermaks, J.; Borovika, A.; Kampf, J. W.; Vedejs, E. Organometallics 2013, 32, 6701.

[33] Cazorla, C.; De Vries, T. S.; Vedejs, E. Org. Lett. 2013, 15, 984.

[34] (a) Doyle, M. P.; McKervey, M. A.; Ye, T. Modern Catalytic Methods for Organic Synthesis with Diazo Compounds, Wiley, New York, 1998.

(b) Dorwald, F. Z. Metal Carbenes in Organic Synthesis, Wiley-VCH, Weinheim, Germany, 1999.

(c) Doyle, M. P. Chem. Rev. 1986, 86, 919 
(d) Doyle, M. P.; Forbes, D. C. Chem. Rev. 1998, 98, 911.

(e) Zhu, S.-F.; Zhou, Q.-L. Nat. Sci. Rev. 2014, 1, 580.

(f) Ford, A.; Miel, H.; Ring, A.; Slattery, C. N.; Maguire, A. R.; McKervey, M. A. Chem. Rev. 2015, 115, 9981.

[35] Bedel, C.; Foucaud, A. Tetrahedron Lett. 1993, 34, 311.

[36] Monnier, L.; Delcros, J.-G.; Carboni, B. Tetrahedron 2000, 56, 6039 .

[37] Imamoto, T.; Yamanoi, Y. Chem. Lett. 1996, 25, 705.

[38] Cheng, Q.-Q.; Zhu, S.-F.; Zhang, Y.-Z.; Xie, X.-L.; Zhou, Q.-L. J. Am. Chem. Soc. 2013, 135, 14094

[39] Cheng, Q.-Q.; Xu, H.; Zhu, S.-F.; Zhou, Q.-L. Acta Chim. Sinica 2015, 73, 326 (in Chinese). (程清卿, 徐唤, 朱守非, 周其林, 化学学报, 2015, 73, 326.)

[40] Li, X.; Curran, D. P. J. Am. Chem. Soc. 2013, 135, 12076.

[41] Allen, T. H.; Curran, D. P. J. Org. Chem. 2016, 81, 2094.

[42] Chen, D.; Zhang, X.; Qi, W.-Y.; Xu, B.; Xu, M.-H. J. Am. Chem. Soc. 2015, 137, 5268.

[43] Hyde, S.; Veliks, J.; Liégault, B.; Grassi, D.; Taillefer, M.; Gouverneur, V. Angew. Chem., Int. Ed. 2016, 55, 3785.

[44] Yang, J.-M.; Li, Z.-Q.; Li, M.-L.; He, Q.; Zhu, S.-F.; Zhou, Q.-L. J. Am. Chem. Soc. 2017, 139, 3784.

[45] For recent examples see: (a) Aramaki, Y.; Omiya, H.; Yamashita, M.; Nakabayashi, K.; Ohkoshi, S.; Nozaki, K. J. Am. Chem. Soc. 2012, 134, 19989

(b) Rosenthal, A. J.; Devillard, M.; Miqueu, K.; Bouhadir, G.; Bourissou, D. Angew. Chem., Int. Ed. 2015, 54, 9198.

(c) Silva Valverde, M. F.; Schweyen, P.; Gisinger, D.; Bannenberg, T.; Freytag, M.; Kleeberg, C.; Tamm, M. Angew. Chem., Int. Ed. $2017,56,1135$.

[46] (a) Roberts, B. P. Chem. Soc. Rev. 1999, 28, 25.

(b) Rablen, P. R. J. Am. Chem. Soc. 1997, 119, 8350.

(c) Walton, J. C. Angew. Chem., Int. Ed. 2009, 48, 1726. (d) Lalevée, J.; Blanchard, N.; Chany, A.-C.; Tehfe, M.-A.; Allonas, X.; Fouassier, J.-P. J. Phys. Org. Chem. 2009, 22, 986.

[47] (a) Barton, D. H. R.; Jacob, M. Tetrahedron Lett. 1998, 39, 1331.

(b) Ueng, S.-H.; Brahmi, M. M.; Derat, É.; Fensterbank, L.; Lacôte, E.; Malacria, M.; Curran, D. P. J. Am. Chem. Soc. 2008, 130, 10082.

[48] (a) Pan, X.; Lalevée, J.; Lacôte, E.; Curran, D. P. Adv. Synth. Catal. 2013, 355, 3522 .

(b) Ueng, S.-H.; Fensterbank, L.; Lacôte, E.; Malacria, M.; Curran, D. P. Org. Biomol. Chem. 2011, 9, 3415.

(c) Pan, X.; Lacôte, E.; Lalevée, J.; Curran, D. P. J. Am. Chem. Soc. 2012, 134, 5669 .

[49] (a) Telitel, S.; Schweizer, S.; Morlet-Savary, F.; Graff, B.; Tschamber, T.; Blanchard, N.; Fouassier, J. P.; Lelli, M.; Lacôte, E.; Lalevée, J. Macromolecules 2013, 46, 43.

(b) Lalevée, J.; Telitel, S.; Tehfe, M. A.; Fouassier, J. P.; Curran, D. P.; Lacôte, E. Angew. Chem., Int. Ed. 2012, 51, 5958.

[50] (a) Pan, X.; Vallet, A.-L.; Schweizer, S.; Dahbi, K.; Delpech, B.; Blanchard, N.; Graff, B.; Geib, S. J.; Curran, D. P.; Lalevée, J.; Lacôte, E. J. Am. Chem. Soc. 2013, 135, 10484.

(b) Telitel, S.; Vallet, A.-L.; Schweizer, S.; Delpech, B.; Blanchard, N.; Morlet-Savary, F.; Graff, B.; Curran, D. P.; Robert, M.; Lacôte, E.; Lalevée, J. J. Am. Chem. Soc. 2013, 135, 16938.

[51] Watanabe, T.; Hirose, D.; Curran, D. P.; Taniguchi, T. Chem.-Eur. J. 2017, 23, 5404.

[52] Ren, S.-C.; Zhang, F.-L.; Qi, J.; Huang, Y.-S.; Xu, A.-Q.; Yan, H.-Y.; Wang, Y.-F. J. Am. Chem. Soc. 2017, 139, 6050.

[53] Solovyev, A.; Chu, Q.; Geib, S. J.; Fensterbank, L.; Malacria, M.; Lacôte, E.; Curran, D. P. J. Am. Chem. Soc. 2010, 132, 15072.

[54] Merling, E.; Lamm, V.; Geib, S. J.; Lacôte, E.; Curran, D. P. Org. Lett. 2012, 14, 2690. 